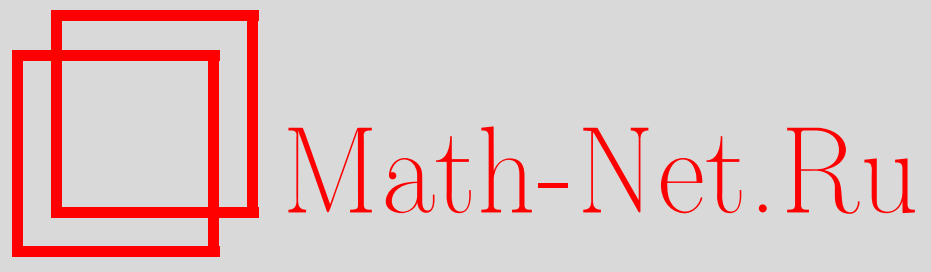

А. А. Хусаинов, Кубические гомологии и размерность Лича свободных частично коммутативных моноидов, Матем. сб., 2008, том 199, номер 12, 129-154

DOI: https://doi.org/10.4213/sm6357

Использование Общероссийского математического портала Math-Net.Ru подразумевает, что вы прочитали и согласны с пользовательским соглашением http://www.mathnet.ru/rus/agreement

Параметры загрузки:

IP : 54.197 .217 .227

26 апреля 2023 г., 09:26:00

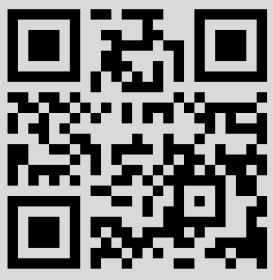




\section{А. А. Хусаинов}

\section{Кубические гомологии и размерность Лича свободных частично коммутативных моноидов}

Работа посвящена проблемам, возникающим при приложении гомологической алгебры к компьютерным наукам. Доказано, что размерность Лича свободного частично коммутативного моноида равна верхней грани мощностей конечных подмножеств его попарно перестановочных образующих. Для произвольного свободного частично коммутативного моноида $M(E, I)$, у которого каждое подмножество попарно перестановочных образующих конечно, и для любой контравариантной натуральной системы $F$ на $M(E, I)$ построено такое полукубическое множество $T(E, I)$ с гомологической системой $\bar{F}$ на нем, что группы гомологий Лича $H_{n}(M(E, I), F)$ изоморфны кубическим группам гомологий $H_{n}(T(E, I), \bar{F})$. Построены также комплексы абелевых групп, позволяющие при дополнительных условиях конечности получить алгоритмы вычисления групп гомологий Лича и гомологий с коэффициентами в правых $M(E, I)$-модулях.

Библиография: 16 названий.

\section{Введение}

Работа посвящена проблемам гомологической алгебры, возникающим при изучении групп гомологий категории состояний параллельной вычислительной системы [1]. Приведем определение моноида, группы гомологий которого исследуются в работе.

Пусть $E$ - множество, $I \subseteq E \times E$ - антирефлексивное симметричное отношение на $E$. Моноид, заданный с помощью множества образующих $E$ и соотношений $a b=b a$, выполненных для всех пар $(a, b) \in I$, обозначается через $M(E, I)$ и называется свободным частично коммутативным. Образующие этого моноида $a, b \in E$, для которых $(a, b) \in I$, мы будем называть независимыми.

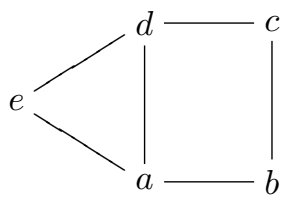

Рис. 1. Пример графа независимости

Моноиду $M(E, I)$ соответствует простой граф с множеством вершин $E$, в котором элементы $a, b \in E$ соединяются ребром в тех случаях, когда $(a, b) \in I$. Этот граф называется графом независимости. Подмножества попарно независимых элементов являются кликами этого графа. На рис. 1 изображен граф моноида $M(E, I)$, заданного множеством $E=\{a, b, c, d\}$ и соотношениями $a b=b a$, $a d=d a, a e=e a, b c=c b, c d=d c, d e=e d$.

(C) А. А. Хусаинов, 2008 
В настоящей работе исследуются размерность Лича, гомологическая размерность и группы гомологий свободных частично коммутативных моноидов. Доказано, что (гомологическая) размерность Лича свободного частично коммутативного моноида равна верхней грани мощностей конечных клик его графа независимости.

Рассматриваемое нами определение свободного частично коммутативного моноида отличается от общепринятого определения [2] тем, что в нем не требуется конечность множества $E$. Если $E$ конечно, то моноид $M(E, I)$ будем называть конечно порожденным. В работе [1] доказано, что гомологическая размерность моноида, не имеющего троек попарно независимых образующих, не больше двух. В [3] была высказана гипотеза о том, что если моноид $M(E, I)$ не содержит $n+1$ попарно независимых образующих, то его гомологическая размерность не больше $n$. Для случая конечного множества $E$ эта гипотеза подтверждена Л. Ю. Поляковой [4]. В настоящей работе эту гипотеза подтверждена для общего случая (теорема 2.12). Кроме того, получен также ответ на поставленный в [1] вопрос о гомологической размерности асинхронной системы переходов (следствие 2.13).

Большое внимание в работе уделено построению комплекса конечной длины для вычисления групп гомологий моноида $M(E, I)$ с коэффициентами в правом $M(E, I)$-модуле. Как известно, группы гомологий $H_{n}(M, A)$ моноида $M$ с коэффициентами в правом $M$-модуле $A$ определяются с помощью комплекса абелевых групп

$$
0 \leftarrow A \stackrel{d_{1}}{\longleftarrow} \bigoplus_{\mu_{1} \in M} A \stackrel{d_{2}}{\longleftarrow} \cdots \stackrel{d_{n-1}}{\longleftarrow} \bigoplus_{\left(\mu_{1}, \ldots, \mu_{n-1}\right) \in M^{n-1}} A \stackrel{d_{n}}{\longleftarrow} \bigoplus_{\left(\mu_{1}, \ldots, \mu_{n}\right) \in M^{n}} A \stackrel{d_{n+1}}{\longleftarrow} \cdots
$$

с дифференциалами

$$
\begin{aligned}
& d_{n}\left(\mu_{1}, \ldots, \mu_{n}, a\right) \\
& \quad=\left(\mu_{2}, \ldots, \mu_{n}, a \cdot \mu_{1}\right)+\sum_{i=1}^{n}(-1)^{i}\left(\mu_{1}, \ldots, \mu_{i-1}, \mu_{i} \mu_{i+1}, \mu_{i+2}, \ldots, \mu_{n}, a\right) .
\end{aligned}
$$

Длина этого комплекса бесконечна и его члены, как правило, содержат бесконечное число слагаемых. Стало быть, он непригоден для вычисления групп гомологий на компьютере. Аналогичная проблема имеет место для гомологий Лича. Для произвольного свободного частично коммутативного моноида $M(E, I)$, в котором каждое подмножество попарно независимых образующих конечно, и для любой контравариантной натуральной системы абелевых групп $F$ на $M(E, I)$ в настоящей работе построены такие полукубическое множество $T(E, I)$ и гомологическая система абелевых групп $\bar{F}$ на нем, что для всех целых $n \geqslant 0$ группы гомологий Лича $H_{n}(M(E, I), F)$ изоморфны группам кубических гомологий $H_{n}(T(E, I), \bar{F})$ (теорема 2.15). Это позволяет построить комплексы конечной длины (теорема 2.16, следствие 2.18), с помощью которых можно вычислять гомологии Лича и гомологии с коэффициентами в правом $M(E, I)$-модуле. В частности, если граф независимости имеет конечное число $n$-клик и натуральная система состоит из свободных конечно порожденных абелевых групп, то для каждого $k>n$ на основе приведения целочисленных матриц к нормальной форме Смита можно построить алгоритм для вычисления $k$-х групп гомологий Лича. 


\section{§ 1. Группы гомологий малых категорий}

В работе будут использоваться следующие обозначения: Set - категория множеств и отображений, $\mathrm{Ab}$ - категория абелевых групп и гомоморфизмов, $\mathbb{Z}$ - аддитивная группа целых чисел, $\mathbb{N}$ - множество неотрицательных целых чисел или свободный моноид $\left\{1, a, a^{2}, \ldots\right\}$, порожденный одним элементом.

Пусть $\mathscr{A}$ - произвольная категория. Класс морфизмов обозначим через $\operatorname{Mor}(\mathscr{A})$, класс объектов - через $\mathrm{Ob}(\mathscr{A})$, дуальную категорию - через $\mathscr{A}^{\mathrm{op}}$. Для любых объектов $A_{1}, A_{2} \in \mathrm{Ob}(\mathscr{A})$ через $\mathscr{A}\left(A_{1}, A_{2}\right)$ будем обозначать множество морфизмов $A_{1} \rightarrow A_{2}$. Функтор, определенный на малой категории и принимающий значения в произвольной категории, называется диаграммой. Для произвольной малой категории $\mathscr{C}$ через $\mathscr{A}^{\mathscr{C}}$ обозначается категория диаграмм $\mathscr{C} \rightarrow \mathscr{A}$. Для произвольного объекта $A \in \mathrm{Ob}(\mathscr{A})$ через $\Delta_{\mathscr{C}} A$ обозначим диаграмму $\mathscr{C} \rightarrow \mathscr{A}$, принимающую постоянные значения, равные $A$ на объектах и $1_{A}$ - на морфизмах.

$\mathrm{B}$ частности, $\Delta_{\mathscr{C}} \mathbb{Z}: \mathscr{C} \rightarrow \mathrm{Ab}$ будет диаграммой, принимающей на объектах значения, равные аддитивной группе целых чисел $\mathbb{Z}$, а на морфизмах - тождественному гомоморфизму $1_{\mathbb{Z}}$. Обозначим ее сокращенно через $\Delta \mathbb{Z}$.

Поскольку гомологии Лича определяются как гомологии категории факторизаций, то в настоящем параграфе приводятся предварительные сведения о гомологиях категорий. Рассматриваются группы гомологий малых категорий $\mathscr{C}$ с коэффициентами в диаграммах $F: \mathscr{C} \rightarrow \mathrm{Ab}$, принимающих значения в категории абелевых групп. Описывается связь между гомологиями малой категории и левыми производными функтора копредела. Предлагаются подходы к оценке гомологической размерности малой категории, основанные на использовании теоремы Оберста об изоморфизме групп $\lim _{\longrightarrow}^{\mathscr{C}^{\mathrm{op}}}(F \circ S) \rightarrow \underset{\lim ^{D^{\circ p}}}{\longrightarrow} F$ в случае строго коинициального функтора $S: \mathscr{C} \rightarrow \mathscr{D}$. Одна из задач, рассматриваемых в работе - оценить размерность свободного частично коммутативного моноида. Эта задача сводится к аналогичной проблеме для коммутативных моноидов и ее решение становится возможным благодаря конструкции категории факторизаций. В $\S 1$ изучается также категория факторизаций и ее гомологии.

\section{1. Гомологии категорий и производные функтора копредела.} Пусть $\mathscr{C}$ - малая категория, $F: \mathscr{C} \rightarrow \mathrm{Ab}$ - диаграмма. Рассмотрим комплекс абелевых групп $C_{*}(\mathscr{C}, F)$, состоящий при $n \geqslant 0$ из прямых сумм

$$
C_{n}(\mathscr{C}, F)=\bigoplus_{c_{0} \rightarrow \cdots \rightarrow c_{n}} F\left(c_{0}\right)
$$

взятых по всем последовательностям $n$ компонируемых морфизмов $c_{0} \rightarrow \cdots \rightarrow$ $c_{n}$ категории $\mathscr{C}$. Элементы прямых слагаемых будем рассматривать как пары $\left(c_{0} \rightarrow \cdots \rightarrow c_{n}, x\right)$, состоящие из индекса $c_{0} \rightarrow \cdots \rightarrow c_{n}$ прямого слагаемого и элемента $x \in F\left(c_{0}\right)$. Положим $C_{n}(\mathscr{C}, F)=0$ при $n<0$. Определим гомоморфизмы $d_{i}^{n}: C_{n}(\mathscr{C}, F) \rightarrow C_{n-1}(\mathscr{C}, F), n>0,0 \leqslant i \leqslant n$, как сопоставляющие каждому элементу

$$
\left(c_{0} \stackrel{\alpha_{1}}{\longrightarrow} c_{1} \stackrel{\alpha_{2}}{\longrightarrow} \cdots \stackrel{\alpha_{n}}{\longrightarrow} c_{n}, x\right) \in \bigoplus_{c_{0} \rightarrow \cdots \rightarrow c_{n}} F\left(c_{0}\right)
$$


элемент

$$
\begin{cases}\left(c_{1} \stackrel{\alpha_{2}}{\longrightarrow} \cdots \stackrel{\alpha_{n}}{\longrightarrow} c_{n}, F\left(c_{0} \stackrel{\alpha_{1}}{\longrightarrow} c_{1}\right)(x)\right), & \text { если } i=0, \\ \left(c_{0} \stackrel{\alpha_{1}}{\longrightarrow} \cdots \stackrel{\alpha_{i-1}}{\longrightarrow} c_{i-1} \stackrel{\alpha_{i+1} \alpha_{i}}{\longrightarrow} c_{i+1} \stackrel{\alpha_{i+2}}{\longrightarrow} \cdots \stackrel{\alpha_{n}}{\longrightarrow} c_{n}, x\right), & \text { если } 1 \leqslant i \leqslant n-1, \\ \left(c_{0} \stackrel{\alpha_{1}}{\longrightarrow} \cdots \stackrel{\alpha_{n-1}}{\longrightarrow} c_{n-1}, x\right), & \text { если } i=n .\end{cases}
$$

Положим $d_{n}=\sum_{i=0}^{n}(-1)^{i} d_{i}^{n}$ при $n>0$ и $d_{n}=0$ при $n \leqslant 0$. Гомоморфизмы $d_{n}$ называются дифференииалами комплекса $C_{*}(\mathscr{C}, F)$.

ОПРедЕЛЕНИЕ 1.1. Абелевы группы $H_{n}(\mathscr{C}, F)=\operatorname{Ker} d_{n} / \operatorname{Im} d_{n+1}$ называются $n$-ми группами гомологий малой категории $\mathscr{C}$ с коэффициентами в диаграмме $F: \mathscr{C} \rightarrow \mathrm{Ab}$.

Например [5; пример 3.2], для категории $p t$, состоящей из единственного объекта 0 и тождественного морфизма, комплекс $C_{*}\left(p t, \Delta_{p t} \mathbb{Z}\right)$ равен

$$
0 \longleftarrow \mathbb{Z} \stackrel{0}{\longleftarrow} \mathbb{Z} \stackrel{1}{\longleftarrow} \mathbb{Z} \stackrel{0}{\longleftarrow} \stackrel{1}{\longleftarrow} \cdots
$$

Его $n$-е группы гомологий равны 0 при $n>0$ и $H_{0}\left(C_{*}(p t, \Delta \mathbb{Z})\right) \cong \mathbb{Z}$.

Хорошо известно [6; приложение 2], что существует изоморфизм левых сателлитов функтора копредела $\lim _{\longrightarrow}^{\mathscr{C}}: \mathrm{Ab}^{\mathscr{C}} \rightarrow \mathrm{Ab}$ и функторов $H_{n}(\mathscr{C},-): \mathrm{Ab}^{\mathscr{C}} \rightarrow$ $\mathrm{Ab}$. Поскольку в категории $\mathrm{Ab}^{\overrightarrow{\mathscr{C}}}$ достаточно проективных и инъективных объектов, то эти сателлиты естественно изоморфны левым производным функтора копредела $\lim ^{\mathscr{C}}: \mathrm{Ab}^{\mathscr{C}} \rightarrow \mathrm{Ab}$. Значения $n$-х сателлитов на $F \in \mathrm{Ab}^{\mathscr{C}}$ будем обозначать через $\lim _{n}^{\mathscr{C}} F$.

В частности, группы $H_{n}\left(\mathscr{C}, \Delta_{\mathscr{C}} \mathbb{Z}\right)$ изоморфны $\lim _{n}^{\mathscr{C}} \Delta_{\mathscr{C}} \mathbb{Z}$. Из теоремы Эйленберга [6; приложение 2] вытекает, что они изоморфны группам гомологий геометрической реализации нерва категории $\mathscr{C}$. Мы будем называть их груnпами целочисленных гомологий малой категории $\mathscr{C}$ и обозначать через $H_{n}(\mathscr{C})$. Например,

$$
H_{n}(p t)= \begin{cases}\mathbb{Z}, & \text { если } n=0, \\ 0, & \text { если } n>0 .\end{cases}
$$

ОПРЕДЕЛЕНИЕ 1.2. Если $H_{n}(\mathscr{C})=0$ для всех $n>0$ и $H_{0}(\mathscr{C}) \cong \mathbb{Z}$, то категория $\mathscr{C}$ называется ацикличной.

Видно, что даже в простейшем случае $\mathscr{C}=p t$ комплекс $C_{*}(\mathscr{C}, F)$ состоит из бесконечного числа ненулевых абелевых групп. В некоторых случаях это компенсируется следующим утверждением, которое доказывается как двойственное к [7; предложение 2.2]. Морфизм $\alpha \in \operatorname{Mor} \mathscr{C}$ называется неединичным, если он не является тождественным. Напомним, что морфизм $\alpha: a \rightarrow b$ называется ретракцией, если существует $\beta: b \rightarrow a$, для которого $\alpha \beta=1_{b}$.

ПредЛОЖЕНИЕ 1.1. Если малая категория $\mathscr{C}$ не содержит неединичных ретракиий, то для любой диаграммы $F: \mathscr{C} \rightarrow \mathrm{Ab}$ группъ $H_{n}(\mathscr{C}, F)$ изоморфнъ группам гомологий подкомплекса $C_{*}^{+}(\mathscr{C}, F) \subseteq C_{*}(\mathscr{C}, F)$, состолщего из сумм

$$
C_{n}^{+}(\mathscr{C}, F)=\bigoplus_{c_{0} \rightarrow \cdots \rightarrow c_{n}} F\left(c_{0}\right), \quad n \geqslant 0,
$$

взятых по последователъностям морфизмов $c_{0} \stackrel{\alpha_{1}}{\longrightarrow} c_{1} \stackrel{\alpha_{2}}{\longrightarrow} \cdots \stackrel{\alpha_{n}}{\longrightarrow} c_{n}, \partial л я$ которьх $\alpha_{i} \neq i d_{c_{i}}$ для всех $1 \leqslant i \leqslant n$. 
В частности, для вычисления групп гомологий категории $\mathscr{C}=p t$ для любого функтора $F: p t \rightarrow$ Аb получаем комплекс $0 \leftarrow F(0) \leftarrow 0 \leftarrow \cdots$, у которого $C_{0}^{+}(\mathscr{C}, F)=F(0)$ и $C_{n}^{+}(\mathscr{C}, F)=0$ при $n>0$.

В дальнейшем нам понадобится следующий

Пример 1.3. Пусть $\Theta$ - категория, состоящая из двух объектов и двух морфизмов $\alpha_{1}: a \rightarrow b$ и $\alpha_{2}: a \rightarrow b$ (кроме тождественных). Пусть $F: \Theta \rightarrow \mathrm{Ab}-$ диаграмма. Вычислим $\lim _{n}^{\Theta} F$. Так как $\lim _{n}^{\Theta}-$ левые производные точного справа функтора $\lim ^{\Theta}, \operatorname{\text {то}}_{\longrightarrow}^{\Theta} F \cong \underline{\lim }^{\Theta} F \cong \operatorname{Coker}\left(F\left(\alpha_{1}\right)-F\left(\alpha_{2}\right)\right)$. Комплекс $C_{*}^{+}(\Theta, F)$ состоит из абелевых групп и гомоморфизмов

$$
0 \longleftarrow F(b) \oplus F(a) \stackrel{d_{1}}{\longleftarrow} \bigoplus_{a \stackrel{\alpha}{\rightarrow} b} F(a) \longleftarrow 0
$$

где $\alpha$ пробегает значения $\alpha_{1}$ и $\alpha_{2}$, а $d_{1}(\alpha, x)=d_{0}^{1}(\alpha, x)-d_{1}^{1}(\alpha, x)$. Дифференциал $d_{1}$ действует на элементах из $\bigoplus_{a \stackrel{\alpha}{\rightarrow} b} F(a)$ как

$$
d_{1}\left(\left(\alpha_{1}, x_{1}\right)+\left(\alpha_{2}, x_{2}\right)\right)=\left(b, F\left(\alpha_{1}\right)\left(x_{1}\right)+F\left(\alpha_{2}\right)\left(x_{2}\right)\right)+\left(a,-x_{1}-x_{2}\right) .
$$

Отсюда

$$
\begin{aligned}
\operatorname{Ker} d_{1} & =\left\{\left(\alpha_{1}, x_{1}\right)+\left(\alpha_{2}, x_{2}\right): x_{1}+x_{2}=0 \& F\left(\alpha_{1}\right)\left(x_{1}\right)+F\left(\alpha_{2}\right)\left(x_{2}\right)=0\right\} \\
& \cong\left\{(x,-x): F\left(\alpha_{1}\right)(x)-F\left(\alpha_{2}\right)(x)=0\right\} \cong \operatorname{Ker}\left(F\left(\alpha_{1}\right)-F\left(\alpha_{2}\right)\right) .
\end{aligned}
$$

Следовательно,

$$
\lim _{\longrightarrow}^{\Theta} F \cong \operatorname{Coker}\left(F\left(\alpha_{1}\right)-F\left(\alpha_{2}\right)\right), \quad \underline{\lim }_{1}^{\Theta} F \cong \operatorname{Ker}\left(F\left(\alpha_{1}\right)-F\left(\alpha_{2}\right)\right) .
$$

1.2. Строго коинициальные функторы. Пусть $S: \mathscr{C} \rightarrow \mathscr{D}$ - функтор из малой категории в произвольную. Для произвольного объекта $d \in \mathrm{Ob}(\mathscr{D})$ слоем (или комма-категорией) $S / d$ называется категория, объектами которой являются пары $(c, \alpha)$, состоящие из объектов $c \in \mathscr{C}$ и морфизмов $\alpha \in \mathscr{D}(S(c), d)$. Морфизмами категории $S / d$ служат тройки $\left(f, \alpha_{1}, \alpha_{2}\right)$, состоящие из морфизмов $f \in \mathscr{C}\left(c_{1}, c_{2}\right), \alpha_{1} \in \mathscr{D}\left(S\left(c_{1}\right), d\right), \alpha_{2} \in \mathscr{D}\left(S\left(c_{2}\right), d\right)$, для которых $\alpha_{2} \circ S(f)=\alpha_{1}$. Забывающим функтором $Q_{d}: S / d \rightarrow \mathscr{C}$ слоя называется функтор, действующий как $Q_{d}(c, \alpha)=c$ на объектах и как $Q_{d}\left(f, \alpha_{1}, \alpha_{2}\right)=f$ - на морфизмах. Если $S$ - полное вложение подкатегории $\mathscr{C} \subseteq \mathscr{D}$, то $S / d$ обозначается через $\mathscr{C} / d$.

ОПредЕЛЕние 1.4. Функтор $S: \mathscr{C} \rightarrow \mathscr{D}$ между малыми категориями называется строго коинициалъным, если для каждого объекта $d \in \mathscr{D}$ слой $S / d$ ацикличен.

Это равносильно тому, что группы целочисленных гомологий $H_{n}(S / d)$ изоморфны группам гомологий точки $H_{n}(p t)$. Кослоем $d / S$ объекта $d \in \mathscr{D}$ над функтором $S: \mathscr{C} \rightarrow \mathscr{D}$ называется категория, объектами которой служат пары $(c, \alpha)$, где $c \in \mathrm{Ob}(\mathscr{C}), \alpha \in \mathscr{D}(d, S(c))$. Ее морфизмы $\left(c_{1}, \alpha_{1}\right) \rightarrow\left(c_{2}, \alpha_{2}\right)$ задаются тройками $\left(f, \alpha_{1}, \alpha_{2}\right)$, состоящими из морфизмов $f \in \mathscr{C}\left(c_{1}, c_{2}\right), \alpha_{1} \in \mathscr{D}\left(d, S\left(c_{1}\right)\right)$, $\alpha_{2} \in \mathscr{D}\left(d, S\left(c_{2}\right)\right)$, удовлетворяющих соотношению $S(f) \circ \alpha_{1}=\alpha_{2}$. 
С помощью [8; теорема 2.3] получаем

ПреДЛОЖеНИЕ 1.2. Пусть $S: \mathscr{C} \rightarrow \mathscr{D}$ - функтор между малыми категориями. Для каждого $n \geqslant 0$ существуют канонические естественные по $F$ гомоморфизмы $\lim _{n}^{\mathscr{C}^{\circ \mathrm{p}}}(F \circ S) \rightarrow \underset{\lim _{n} \mathscr{D}^{\mathrm{op}}}{\longrightarrow} F$. Для того чтобы эти гомоморфизмы были изоморбизмами для каждого $n \geqslant 0$ и $\vec{F}: \mathscr{D}^{\mathrm{op}} \rightarrow \mathrm{Ab}$, необходимо u достаточно, чтобы бунктор $S$ был строго коинициальным.

1.3. Категория факторизаций. Пусть $\mathscr{C}$ - малая категория. Ее категория факторизаций $\mathfrak{F} \mathscr{C}$ (см. [9]) имеет множество объектов $\operatorname{Ob}(\mathfrak{F} \mathscr{C})=\operatorname{Mor}(\mathscr{C})$, состоящее из всех морфизмов категории $\mathscr{C}$. Ее множества морфизмов $\mathfrak{F} \mathscr{C}(\alpha, \beta)$ между $\alpha, \beta \in \mathrm{Ob}(\mathfrak{F} \mathscr{C})$ состоят из пар $(f, g)$ морфизмов $f, g \in \operatorname{Mor}(\mathscr{C})$, для которых композиция $g \circ \alpha \circ f$ определена (рис. 2) и равна $g \circ \alpha \circ f=\beta$. Композиция морфизмов $\alpha \stackrel{\left(f_{1}, g_{1}\right)}{\longrightarrow} \beta$ и $\beta \stackrel{\left(f_{2}, g_{2}\right)}{\longrightarrow} \gamma$ определяется как $\alpha \stackrel{\left(f_{1} \circ f_{2}, g_{2} \circ g_{1}\right)}{\longrightarrow} \gamma$. Тождественный морфизм объекта $a \stackrel{\alpha}{\longrightarrow} b$ категории $\mathfrak{F} \mathscr{C}$ состоит из пары тождественных морфизмов $\alpha \stackrel{\left(1_{a}, 1_{b}\right)}{\longrightarrow} \alpha$.

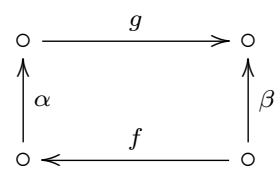

Рис. 2. Морфизм категории факторизаций $\alpha \stackrel{(f, g)}{\longrightarrow} \beta$

Лемма 1.3. Для любой категории $\mathscr{C}$ существует изоморфизм $\mathfrak{F}\left(\mathscr{C}^{\mathrm{op}}\right) \cong$ $\mathfrak{F} \mathscr{C}$.

ДокАзАтЕЛьСтво. Категория $\mathscr{C}^{\text {ор }}$ имеет класс объектов $\mathrm{Ob}\left(\mathscr{C}^{\mathrm{op}}\right)=\mathrm{Ob}(\mathscr{C})$. Ее морфизмы можно рассматривать как символы $\alpha^{\mathrm{op}}$, где $\alpha$ пробегает все морфизмы категории $\mathscr{C}$. Композиция морфизмов в $\mathscr{C}^{\text {ор }}$ определена по закону $\alpha^{\text {op }} \circ \beta^{\text {op }}=(\beta \circ \alpha)^{\text {op }}$. Искомый изоморфизм категорий $\mathfrak{F}\left(\mathscr{C}^{\text {op }}\right) \cong \mathfrak{F} \mathscr{C}$ осуществляет отображение, действующее на объектах как $\alpha \mapsto \alpha^{\text {op }}$, а на морфизмах как $(\alpha \stackrel{(f, g)}{\longrightarrow} \beta) \longmapsto\left(\alpha^{\mathrm{op}} \stackrel{\left(g^{\mathrm{op}}, f^{\mathrm{op}}\right)}{\longrightarrow} \beta^{\mathrm{op}}\right)$.

Для $\alpha \in \operatorname{Mor}(\mathscr{C})$ комма-категория $\mathfrak{F} \mathscr{C} / \alpha$ состоит из троек $(x, \beta, y)$, для которых $y \circ \beta \circ x=\alpha$. Морфизмы в ней задаются парами $(v, w)$, удовлетворяющими соотношениям $w \circ \beta \circ v=\beta^{\prime}, v \circ x^{\prime}=x$ и $y^{\prime} \circ w=y$. Поскольку $\mathfrak{F} \mathscr{C} / \alpha \cong \mathfrak{F}\left(\mathscr{C}^{\text {op }}\right) / \alpha^{\text {op }}$, то получим

СЛЕДСТВиЕ 1.4. Пусть $\mathscr{C}-$ категория, $\alpha-e$ морфизм. Тогда категория $\mathfrak{F} \mathscr{C} / \alpha$ будет изоморфна категории, обгектами которой являются тройки морфизмов $(x, \beta, y)$, композичия которых определена и равна $x \circ \beta \circ y=\alpha$, а морбизмы $(x, \beta, y) \rightarrow\left(x^{\prime}, \beta^{\prime}, y^{\prime}\right)$ задаются коммутативными диаграммами, показанными на рис. 3 .

В работе [9] были определены когомологии категории с коэффициентами в натуральной системе. Согласно [9; теорема 4.4] они естественно изоморфны 


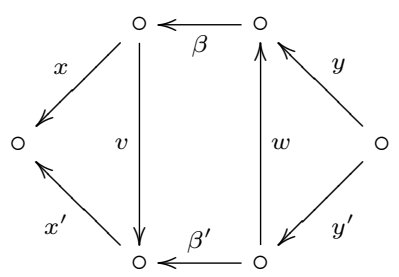

Рис. 3. Морфизм комма-категории $\mathfrak{F}\left(\mathscr{C}^{\mathrm{op}}\right) / \alpha$

правым производным $\lim _{\mathfrak{F} \mathscr{C}}^{n}$ функтора предела. Введем двойственное определение.

Пусть $\mathscr{C}$ - произвольная малая категория, $\mathfrak{F} \mathscr{C}$ - ее категория факторизаций. Контравариантной натуралъной системой на $\mathscr{C}$ называется диаграмма $F:(\mathfrak{F} \mathscr{C})^{\text {op }} \rightarrow \mathrm{Ab}$

ОПРЕДЕЛЕНИЕ 1.5. Группами гомологий малой категории $\mathscr{C}$ с коэффициентами в контравариантной натуральной системе $F$ на $\mathscr{C}$ называются абелевы группы $\lim _{n}^{(\mathfrak{F} \mathscr{C})^{\mathrm{op}}} F$.

Поскольку категория факторизаций имеет сложное строение, то можно попытаться найти ее коинициальные подкатегории. Для этого необходимо изучить комма-категории $\mathfrak{F} \mathscr{C} / \alpha$. Категория называется категорией с сокращениями, если каждый ее морфизм является эпиморфизмом и мономорфизмом. Выполнение этого условия равносильно истинности импликаций $\alpha \gamma=\beta \gamma \Longrightarrow$ $\alpha=\beta$ и $\gamma \alpha=\gamma \beta \Longrightarrow \alpha=\beta$ для всех $\alpha, \beta, \gamma \in \mathscr{C}$.

Докажем утверждение, полезное при изучении гомологий категорий с сокращениями.

Напомним, что предупорядоченное множество можно определить как малую категорию $\mathscr{C}$, у которой множество морфизмов $\mathscr{C}(a, b)$ для любых $a, b \in \mathrm{Ob} \mathscr{C}$ имеет не более одного элемента. Положим $a \leqslant b$, если $\mathscr{C}(a, b) \neq \varnothing$. Если для этой категории $\mathscr{C}$ верна импликация $\mathscr{C}(a, b) \neq \varnothing \mathscr{\&} \mathscr{C}(b, a) \neq \varnothing \Longrightarrow a=b$, то отношение предпорядка будет антисимметричным и категория превратится в частично упорядоченное множество.

Лемма 1.5. Если $\mathscr{C}$ - малая категория с сокращениями, не содержащая неединичных ретракиий, то для любого ее морфизма $\alpha \in \mathrm{Ob}(\mathfrak{F} \mathscr{C})$ комма-категория $\mathfrak{F} \mathscr{C} / \alpha$ является частично упорядоченным множеством.

ДокАЗАтЕльСтво. Объектами категории $\mathfrak{F} \mathscr{C} / \alpha$ для произвольного морфизма $\alpha: a \rightarrow b$ являются тройки морфизмов $(x, w, y)$, композиция которых $y \circ w \circ x$ равна $\alpha$. Рассмотрим любые два объекта этой категории и два морфизма между ними $(x, w, y) \underset{\left(u_{2}, v_{2}\right)}{\stackrel{\left(u_{1}, v_{1}\right)}{\rightrightarrows}}\left(x^{\prime}, w^{\prime}, y^{\prime}\right)$. Эти морфизмы задаются с помощью коммутативной диаграммы, показаной на рис. 4. Из равенств $y^{\prime} v_{1}=y=y^{\prime} v_{2}$ следует, что $v_{1}=v_{2}$, а из равенств $u_{1} x^{\prime}=x=u_{2} x^{\prime}$ следует, что $u_{1}=u_{2}$. Отсюда $u_{1}=u_{2}$ и $v_{1}=v_{2}$. Следовательно, любые два морфизма $(x, w, y) \rightarrow\left(x^{\prime}, w^{\prime}, y^{\prime}\right)$ совпадают, откуда вытекает, что $\mathfrak{F} \mathscr{C} / \alpha-$ предупорядоченное множество. Если в $\mathscr{C}$ 
нет неединичных ретракций, то любые два морфизма $(x, w, y) \underset{\left(u_{2}, v_{2}\right)}{\stackrel{\left(u_{1}, v_{1}\right)}{\rightleftarrows}}\left(x^{\prime}, w^{\prime}, y^{\prime}\right)$ приводят к равенствам $u_{1} x^{\prime}=x, u_{2} x=x^{\prime}, y^{\prime} v_{1}=y, y v_{2}=y^{\prime}$. Эти равенства дают соотношения $u_{1} u_{2} x=x, y v_{2} v_{1}=y$. Поскольку $\mathscr{C}$ допускает сокращения, то получаем, что $u_{1} u_{2}$ и $v_{2} v_{1}$ - тождественные морфизмы. Если в $\mathscr{C}$ нет неединичных ретракций, то отсюда вытекает, что $u_{1}, u_{2}, v_{1}$ и $v_{2}$ - тождественные морфизмы. Следовательно, отношение предпорядка на $\mathfrak{F} \mathscr{C} / \alpha$ антисимметрично и $\mathfrak{F} \mathscr{C} / \alpha$ - частично упорядоченное множество.

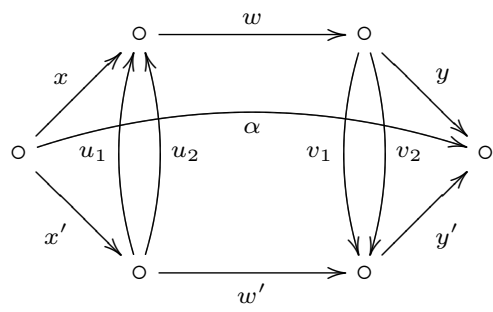

Рис. 4. Пара морфизмов категории $\mathfrak{F} \mathscr{C} / \alpha$

Для произвольной малой категории $\mathscr{C}$ рассмотрим функтор $\mathfrak{F} \mathscr{C} \stackrel{t}{\rightarrow} \mathscr{C}$, сопоставляющий каждой стрелке $a \rightarrow b$ ее конец $b$, каждому морфизму $\alpha \stackrel{(f, g)}{\longrightarrow} \beta-$ морфизм $g: t(\alpha) \rightarrow t(\beta)$. Следующее утверждение доказано в [7; 2$]$, но не сформулировано там в явном виде.

ЛЕмма 1.6. Функтор $\mathfrak{F} \mathscr{C} \stackrel{t}{\rightarrow} \mathscr{C}$ строго коинициален.

ДокАЗАТЕЛЬСтво. Для всякого $c \in \mathscr{C}$ категория $t / c$ содержит полную подкатегорию, состоящую из объектов вида $\left(\beta, 1_{c}\right)$. Эта подкатегория изоморфна $(\mathscr{C} / c)^{\text {ор }}$. Для каждого объекта $(\alpha, x)$ категории $t / c$ определен морфизм $(\alpha, x) \rightarrow\left(x \circ \alpha, 1_{c}\right)$, через который единственным образом пропускается всякий морфизм $(\alpha, x) \rightarrow\left(\beta, 1_{c}\right)$ категории $t / c$. Это свойство универсальности показывает, что полное вложение $(\mathscr{C} / c)^{\mathrm{op}} \subseteq t / c$ обладает левым сопряженным. Отсюда следует, что функтор вложения $\mathscr{C} / c \subseteq(t / c)^{\text {ор }}$ строго коинициален. Следовательно, $\lim _{\longrightarrow}^{t / c}(\Delta \mathbb{Z}) \circ \sigma \cong \lim _{n}^{(\mathscr{C} / c)^{\text {ор }}}(\Delta \mathbb{Z})$. Стало быть, $H_{q}(t / c)=0$ при $q>0$ и $H_{0}(t / c) \cong{ }_{\mathbb{Z}}^{n}$.

С помощью предложения 1.2 получаем

СледСТВИЕ 1.7. Для произвольной диаграммы $F: \mathscr{C}^{\mathrm{op}} \rightarrow \mathrm{Ab}$ имеют место изоморфизмы $\lim _{\mathfrak{F}} \mathscr{F}^{\mathrm{OP}} F \circ t^{\mathrm{op}} \cong \lim _{n}^{\mathscr{C}^{\mathrm{OP}}} F$ для всех $n \geqslant 0$.

1.4. Гомологическая размерность малых категорий. Пусть $\mathbb{N}$ - множество неотрицательных целых чисел. Будем рассматривать его как подмножество множества $\{-1\} \cup \mathbb{N} \cup\{\infty\}$ с обычным отношением порядка между целыми числами и отношением $n \leqslant \infty$ для всех $n=-1,0,1,2, \ldots$.

ОПРЕДЕЛЕНИЕ 1.6. Гомологической размерностъю һd $\mathscr{C}$ малой категории $\mathscr{C}$ называется точная верхняя грань в $\{-1\} \cup \mathbb{N} \cup\{\infty\}$ множества чисел $n \in \mathbb{N}$, для которых функторы $\lim _{n}^{\mathscr{C}}: \mathrm{Ab}^{\mathscr{C}} \rightarrow \mathrm{Ab}$ не равны нулю. 
Из предложения 1.1 вытекает

СлЕДСтвиЕ 1.8. Если малая категория $\mathscr{C}$ не содержит неединичных ретракций и длина $m$ произволъной последовательности неединичных морфизмов $c_{0} \stackrel{\alpha_{1}}{\longrightarrow} \cdots \stackrel{\alpha_{m}}{\longrightarrow} c_{m}$ не больше, чем $n$, mo hd $\mathscr{C} \leqslant n$.

$\mathrm{B}$ частности, hd $p t=0$. Гомологическая размерность категории $\Theta$ из примера 1.3 равна 1 .

Предложение 1.2 дает важное

СлеДСтвиЕ 1.9. Если существует строго коинициальный функтор $S$ : $\mathscr{C} \rightarrow \mathscr{D}$ между малыми категориями, mо hd $\mathscr{C}^{\mathrm{op}} \geqslant$ hd $\mathscr{D}^{\mathrm{op}}$.

Открытой подкатегорией категории $\mathscr{C}$ называется полная подкатегория $\mathscr{D} \subseteq \mathscr{C}$, которая вместе с любым объектом $d \in \mathscr{D}$ содержит все объекты $c \in \mathscr{C}$, допускающие морфизмы $d \rightarrow c$. Подкатегория $\mathscr{D} \subseteq \mathscr{C}$ называется замкнутой, если $\mathscr{D}^{\mathrm{op}}$ - открытая подкатегория в $\mathscr{C}^{\mathrm{op}}$.

Пусть $\mathscr{D} \subseteq \mathscr{C}$ - замкнутая подкатегория. Рассмотрим функтор ограничения $\mathrm{Ab}^{\mathscr{C}^{\mathrm{op}}} \rightarrow \mathrm{Ab}^{\mathscr{D}^{\mathrm{op}}},\left.G \mapsto G\right|_{\mathscr{D} \text { ор }}$. Он имеет левый сопряженный $\mathrm{Ab}^{\mathscr{D}^{\mathrm{op}}} \rightarrow \mathrm{Ab}^{\mathscr{C}^{\mathrm{op}}}$, cопоставляющий каждой диаграмме $F \in \mathrm{Ab}^{\mathscr{D}^{\circ}}$ диаграмму $F_{\mathscr{D}}$, полученную из $F$ добавлением нулей: $\left.F_{\mathscr{D}}\right|_{\mathscr{D} \text { ор }}=F$ и $F_{\mathscr{D}}(c)=0$ при $c \notin \mathrm{Ob}(\mathscr{D})$. Легко видеть, что оба функтора точны и функтор добавления нулей сопряжен слева к функтору ограничения.

Лемма 1.10. Пусть $\mathscr{D} \subseteq \mathscr{C}-$ замкнутая подкатегория. Тогда для любой диаграммы $F \in \mathrm{Ab}^{\mathscr{D}^{\mathrm{op}}}$ верно $\lim _{\mathscr{C}^{\mathrm{op}}} F_{\mathscr{D}} \cong \underline{\lim }_{\mathfrak{D}}^{\mathrm{op}^{\mathrm{P}}} F$.

ДокАЗАТЕЛЬСтво. Рассмотрим произвольную проективную резольвенту диаграммы $F \in \mathrm{Ab}^{\mathscr{D} \mathrm{p}}$

$$
0 \leftarrow F \leftarrow F_{0} \leftarrow F_{1} \leftarrow F_{2} \leftarrow \cdots
$$

Так как $\lim _{n}^{\mathscr{D}^{\circ}}$ - производные функтора копредела, то применение к этой резольвенте функтора копредела приводит к комплексу $\lim ^{\mathscr{D}^{\circ \mathrm{p}}} F_{*}$ с группами гомологий $H_{n}\left({\stackrel{\lim }{\mathscr{D}^{\mathrm{op}}}} F_{*}\right) \cong{\underset{\lim }{D^{\mathrm{op}}}}_{n} F$. С другой стороны, функтор добавления нулей переводит эту резольвенту в проективную резольвенту $F_{* \mathscr{D}}$ функтора $F_{\mathscr{D}}$. Так как $\lim ^{\mathscr{C}^{\mathrm{op}}} F_{\mathscr{D}} \cong \lim ^{\mathscr{D} \mathrm{op}} F$, то комплекс $\underline{\lim }^{\mathscr{C}^{\mathrm{op}}} F_{* \mathscr{D}}$ будет изоморфен комплексу $\underset{\lim ^{\circ}}{\longrightarrow} F_{*}$. Стало быть, изоморфны группы гомологий этих комплексов, $\lim _{n}^{\mathscr{C}^{\mathrm{op}}} F_{\mathscr{D}} \cong \lim _{\longrightarrow}^{\mathscr{D}^{\mathrm{op}}} F$.

ЛЕмма 1.11. Для произвольной открытой подкатегории $\mathscr{D} \subseteq \mathscr{C}$ имеет место неравенство hd $\mathscr{D} \leqslant \mathrm{hd} \mathscr{C}$. Если $\bigcup_{j \in J} \mathscr{C}_{j}$ - объединение открытых подкатегорий малой категории $\mathscr{C}$, то

$$
\text { hd } \bigcup_{j \in J} \mathscr{C}_{j}=\sup _{j \in J}\left\{\operatorname{hd} \mathscr{C}_{j}\right\} .
$$

ДокАЗАТЕЛЬство. Для любого объекта $a \in \mathscr{C}$ обозначим через $\mathscr{C}^{a} \subseteq \mathscr{C}$ полную подкатегорию категории $\mathscr{C}$, объекты которой $b \in \mathscr{C}^{a}$ допускают морфизмы $a \rightarrow b$. Согласно Митчелу [10; следствие 10] hd $\mathscr{C}=\sup _{a \in \operatorname{Ob} \mathscr{C}}\left\{\right.$ hd $\left.\mathscr{C}^{a}\right\}$. Если $\mathscr{D}$ - открытая подкатегория, то $\mathscr{D}=\bigcup_{d \in \mathrm{Ob}(\mathscr{D})} \mathscr{C}^{d}$. Отсюда hd $\mathscr{D} \leqslant$ hd $\mathscr{C}$. 
Если подкатегории $\mathscr{C}_{j}$ открыты, то для каждого $a \in \mathscr{C}_{j}$ верно $\mathscr{C}^{a}=\mathscr{C}_{j}^{a}$ и второе утверждение вытекает из соотношения

$$
\sup _{j}\left\{\sup _{a \in \mathrm{Ob} \mathscr{C}_{j}}\left\{\operatorname{hd} \mathscr{C}^{a}\right\}\right\}=\sup _{a \in \cup_{j \in J} \mathrm{Ob} \mathscr{C}_{j}}\left\{\mathrm{hd} \mathscr{C}^{a}\right\} .
$$

1.5. Ацикличные частично упорядоченные множества. Напомним, что малая категория называется ацикличной, если ее целочисленные гомологии изоморфны гомологиям точки. Нахождение строго коинициальных функторов тесно связано со свойством ацикличности. Найдем одно из простейших условий ацикличности объединения частично упорядоченных множеств.

Лемма 1.12. Пусть $X$ - частично упорядоченное множество, $V u W-$ его такие замкнутые подмножества, что $X=V \cup W$. Тогда если $V, W u$ $V \cap W-$ ацикличны, то $X-$ ациклично.

Доказательство. Пусть $C_{*}(X)=C_{*}\left(X, \Delta_{X} \mathbb{Z}\right)$. Рассмотрим цепной гомоморфизм $C_{*}(X) \rightarrow C_{*}(p t)$. Его ядро обозначим через $\widetilde{C}_{*}(X)$. Группы гомологий $H_{q}\left(\widetilde{C}_{*}(X)\right)$ называются приведенными группами гомологий и обозначаются через $\widetilde{H}_{q}(X)$. Легко видеть, что $X$ будет ацикличным тогда и только тогда, когда $\widetilde{H}_{q}(X)=0$ для всех $q \geqslant 0$. Пусть $X^{\text {ор }}-$ двойственное частично упорядоченное множество. Тогда $V^{\text {op }}$ и $W^{\text {op }}$ - открытые подмножества и $X^{\mathrm{op}}=V^{\mathrm{op}} \cup W^{\mathrm{op}}$. Из точной последовательности

$$
0 \rightarrow C_{*}\left(V^{\mathrm{op}} \cap W^{\mathrm{op}}\right) \rightarrow C_{*}\left(V^{\mathrm{op}}\right) \oplus C_{*}\left(W^{\mathrm{op}}\right) \rightarrow C_{*}\left(X^{\mathrm{op}}\right) \rightarrow 0
$$

с помощью $(3 \times 3)$-леммы получаем точную последовательность

$$
0 \rightarrow \widetilde{C}_{*}\left(V^{\mathrm{op}} \cap W^{\mathrm{op}}\right) \rightarrow \widetilde{C}_{*}\left(V^{\mathrm{op}}\right) \oplus \widetilde{C}_{*}\left(W^{\mathrm{op}}\right) \rightarrow \widetilde{C}_{*}\left(X^{\mathrm{op}}\right) \rightarrow 0 .
$$

Соответствующая длинная точная последовательность групп гомологий приводит к изоморфизмам $\widetilde{H}_{q}\left(X^{\text {op }}\right)=0$ для всех $q \geqslant 0$.

Применяя индукцию по числу покрывающих множеств, легко получить

СлЕДСтвиЕ 1.13. Пусть $X=\bigcup_{i=1}^{n} W_{i}$ - покрытие частично упорядоченного множества ацикличными замкнутыми подмножествами. Тогда если для любого подмножества $\left\{i_{1}, i_{2}, \ldots, i_{k}\right\} \subseteq\{1,2, \ldots, n\}$ подмножество $W_{i_{1}} \cap$ $W_{i_{2}} \cap \cdots \cap W_{i_{k}}$ ациклично, то $X$ ачиклично.

ЛЕмма 1.14 (Формула Кюннета). Пусть $\mathscr{C}$ и $\mathscr{D}$ - малые категории. Для любого $n \geqslant 0$ группа $H_{n}(\mathscr{C} \times \mathscr{D})$ изоморфна

$$
\left(\bigoplus_{p+q=n} H_{p}(\mathscr{C}) \otimes H_{q}(\mathscr{D})\right) \oplus\left(\bigoplus_{p+q=n-1} \operatorname{Tor}\left(H_{p}(\mathscr{C}), H_{q}(\mathscr{D})\right)\right) .
$$

ДокАЗАТЕЛЬство. Нерв категории $\mathscr{C} \times \mathscr{D}$ изоморфен произведению нервов. По теореме Эйленберга-Зильбера [11; гл. VIII, теорема 8.1] цепные комплексы $C_{*}(\mathscr{C} \times \mathscr{D}, \Delta \mathbb{Z})$ и $C_{*}(\mathscr{C}, \Delta \mathbb{Z}) \otimes C_{*}(\mathscr{D}, \Delta \mathbb{Z})$ гомотопически эквивалентны. Искомое утверждение вытекает из формулы Кюннета для тензорного произведения комплексов [11; гл. V, теорема 10.2].

СлЕДСТвИЕ 1.15. Пусть $X$ и $Y$ - частично упорядоченные множества. Если $X$ и $Y$ ачикличнь, то их произведение $X \times Y$ ациклично. 
1.6. Гомологии полукубических множеств. Одно из приложений теории производных функторов копредела - построение теории гомологий полукубических множеств с коэффициентами в гомологических системах коэффициентов [5]. Эта теория обобщает относительные гомологии кубических подмножеств евклидовых пространств [12] и гомологии Губо полукубических множеств [13]. Нам понадобятся результаты, доказательство которых дано в [5].

Полукубическим множеством $X=\left(X_{n}, \partial_{i}^{n, \varepsilon}\right)$ называется последовательность множеств $\left(X_{n}\right)_{n \in \mathbb{N}}$ и семейство отображений $\partial_{i}^{n, \varepsilon}: X_{n} \rightarrow X_{n-1}$, определенных при $1 \leqslant i \leqslant n, \varepsilon \in\{0,1\}$, и удовлетворяющих условию коммутативности показанных на рис. 5 диаграмм для всех $\alpha, \beta \in\{0,1\}, n \geqslant 2$ и $1 \leqslant i<j \leqslant n$.

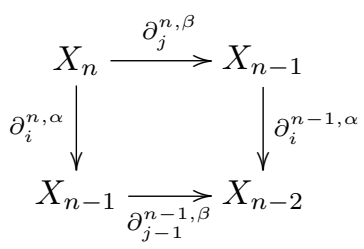

Рис. 5. Коммутативная диаграмма граничных отображений

Пусть $\square_{+}-$категория, состоящая из конечных частично упорядоченных множеств $\mathbb{I}^{n}=\{0,1\}^{n}$, равных декартовой степени линейно упорядоченного множества $\mathbb{I}=\{0,1\}$. Морфизмы этой категории определяются как возрастающие отображения частично упорядоченных множеств, допускающих разложение в композицию отображений вида $\delta_{i}^{k, \varepsilon}: \mathbb{I}^{k-1} \rightarrow \mathbb{I}^{k}$, где

$$
\delta_{i}^{k, \varepsilon}\left(x_{1}, \ldots, x_{k-1}\right)=\left(x_{1}, \ldots, x_{i-1}, \varepsilon, x_{i}, \ldots, x_{k-1}\right), \quad \varepsilon \in \mathbb{I}, \quad 1 \leqslant i \leqslant k .
$$

Каждый морфизм этой категории $f: \mathbb{I}^{m} \rightarrow \mathbb{I}^{n}$ допускает каноническое разложение $f=\delta_{j_{n-m}}^{n, \varepsilon_{n-m}} \cdots \delta_{j_{1}}^{m+1, \varepsilon_{1}}, 1 \leqslant j_{1}<\cdots<j_{n-m} \leqslant n$. Поэтому полукубическое множество можно определить как функтор $X: \square_{+}^{\text {op }} \rightarrow$ Set, принимающий значения $X\left(\mathbb{I}^{n}\right)=X_{n}$ на объектах и $X(f)=\partial_{j_{1}}^{m+1, \varepsilon_{1}} \cdots \partial_{j_{n-m}}^{n, \varepsilon_{n-m}}$ - на морфизмах $f=\delta_{j_{n-m}}^{n, \varepsilon_{n-m}} \cdots \delta_{j_{1}}^{m+1, \varepsilon_{1}}$. Определяя морфизмы как естественные преобразования, получаем категорию полукубических множеств $\mathrm{Set}_{+}^{\square_{+}^{\text {op }}}$.

Пусть $h_{*}: \square_{+} \rightarrow$ Set $^{\square_{+}^{\text {op }}}$ - вложение Ионеды. Для произвольного $m \geqslant 0$ и $\sigma \in X_{m}$ обозначим через $\tilde{\sigma}: h_{\mathbb{I}} \rightarrow X$ естественное преобразование, соответствующее элементу $\sigma \in X_{m}$ при биекции

$$
\operatorname{Set}_{+}^{\square_{+}^{\mathrm{op}}}\left(h_{\mathbb{I}^{m}}, X\right) \cong X_{m},
$$

вытекающей из леммы Ионеды. Поскольку $h_{*}-$ полное вложение, то каждый объект $\left(\mathbb{I}^{m}, \tilde{\sigma}: h_{\mathbb{I} m} \rightarrow X\right)$ определен вторым элементом пары - морфизмом $\tilde{\sigma}: h_{\mathbb{I} m} \rightarrow X$. Поэтому можно считать, что объектами категории $h_{*} / X$ являются морфизмы $\widetilde{\sigma}: h_{\mathbb{I} m} \rightarrow X$, а морфизмами - коммутативные треугольники, показанные на рис. 6 . Согласно [14] категория $h_{*} / X$ будет изоморфна категории $\square_{+} / X$, объектами которой являются элементы $\sigma \in \coprod_{n \geqslant 0} X_{n}$, а морфизмы между $\sigma \in X_{n}$ и $\tau \in X_{m}$ задаются такими тройками $(\alpha, \sigma, \tau)$, что $\alpha \in \square_{+}\left(\mathbb{I}^{m}, \mathbb{I}^{n}\right)$ и $X(\alpha)(\tau)=\sigma$. Обозначим эти тройки через $\alpha: \sigma \rightarrow \tau$. 


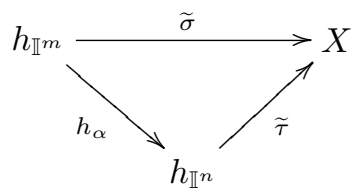

Рис. 6. Морфизм категории $h_{*} / X$

Гомологической системой абелевых групп на $X$ называется произвольный функтор $F:\left(\square_{+} / X\right)^{\text {op }} \rightarrow$ Ab. Рассмотрим абелевы группы

$$
C_{n}(X, F)=\bigoplus_{\sigma \in X_{n}} F(\sigma) .
$$

Определим гомоморфизмы $d_{i}^{n, \varepsilon}: C_{n}(X, F) \rightarrow C_{n-1}(X, F)$ как делающие коммутативными диаграммы на рис. 7 . Пусть $C_{*}(X, F)$ - комплекс, состоящий из групп $C_{n}(X, F)=\bigoplus_{\sigma \in X_{n}} F(\sigma)$ и дифференциалов $d_{n}=\sum_{i=1}^{n}(-1)^{i}\left(d_{i}^{n, 1}-d_{i}^{n, 0}\right)$.

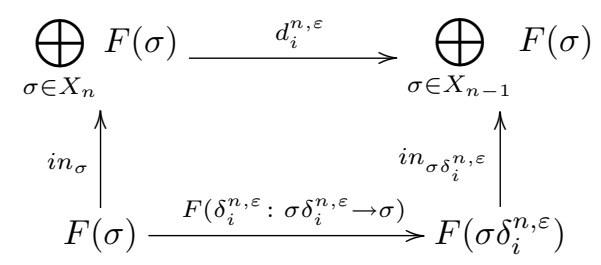

Рис. 7. Определение граничных гомоморфизмов

В работе [5] доказана следующая

Теорема 1.16. Значения сателлитов функтора копредела $\lim _{n}^{\left(\square_{+} / X\right)^{\mathrm{op}}} F$ естественно изоморфны $n$-м группам гомологий комплекса $C_{*}(X, F)$.

\section{§ 2. Гомологии Лича свободных частично коммутативных моноидов}

В этом параграфе для произвольного моноида введено определение его групп гомологий Лича с коэффициентами в контравариантной натуральной системе. Вычислены группы гомологий Лича бесконечного циклического моноида $\mathbb{N}=\left\{1, a, a^{2}, a^{3}, \ldots\right\}$. Доказано, что полная подкатегория категории факторизаций, состоящая из конечных произведений различных попарно независимых образующих, строго коинициальна. Отсюда следует, что гомологии Лича частично коммутативного моноида изоморфны гомологиям этой подкатегории.

\section{1. Категория факторизаций бесконечного циклического монои-} да. Пусть $M$ - произвольный моноид. Будем рассматривать его как категорию, имеющую единственный объект. Пусть $\mathfrak{F} M-$ категория факторизаций 
этого моноида. Наша цель - найти инициальную подкатегорию категории факторизаций моноида $\mathbb{N}=\left\{1, a, a^{2}, \ldots\right\}$. Как пример приложения, мы получим формулу для гомологий Лича моноида $\mathbb{N}$.

В работе Лича [15] рассматривались когомологии моноида с коэффициентами в функторе на категории факторизаций. Мы будем рассматривать следующие гомологии.

ОПРЕДЕЛЕНИЕ 2.1. Гомологиями Лича $H_{n}(M, F)$ моноида $M$ с коэффициентами в контравариантной натуральной системе $F:(\mathfrak{F} M)^{\mathrm{op}} \rightarrow \mathrm{Ab}$ называются группы $\lim _{n}^{(\mathfrak{F} M)^{\mathrm{op}}} F$.

Лемма 2.1. Пусть $T=\{1, a\}-$ подмножество моноида $\mathbb{N}=\left\{1, a, a^{2}, \ldots\right\}$, $\mathfrak{F T} \subset \mathfrak{F N}$ - полная подкатегория категории факторизаций этого моноида, множество обгектов которой совпадает с T. Тогда вложение $\mathfrak{F} \subset \subset \mathfrak{F}$ строго коинициально.

ДокАЗАТЕЛЬСтво. Категория $\mathfrak{F T}$ будет состоять из двух объектов и двух морфизмов $1 \stackrel{(1, a)}{\longrightarrow} a, 1 \stackrel{(a, 1)}{\longrightarrow} a$, кроме тождественных. Легко видеть, что для каждого целого числа $p \geqslant 0$ объекты слоя $\mathfrak{F} T / a^{p}$ можно разбить на два класса:

- $1 \stackrel{\left(a^{s}, a^{t}\right)}{\longrightarrow} a^{p}$, где $s+t=p, s \geqslant 0, t \geqslant 0$ и $p \geqslant 0$,

- $a \stackrel{\left(a^{s}, a^{t}\right)}{\longrightarrow} a^{p}$, где $s+t=p-1, s \geqslant 0, t \geqslant 0$ и $p \geqslant 1$.

Область и кообласть любого неединичного морфизма категории $\mathfrak{F} T / a^{p}$ принадлежат различным классам. По лемме 1.5 категория $\mathfrak{F} T / a^{p}$ является частично упорядоченным множеством как полная подкатегория в $\mathfrak{F} \mathbb{N} / a^{p}$. Легко видеть, что категория $\mathfrak{F} T / a^{p}$ изоморфна частично упорядоченному множеству

$$
\begin{gathered}
\left(1,1, a^{p}\right)<\left(1, a, a^{p-1}\right)>\left(a^{1}, 1, a^{p-1}\right)<\cdots \\
\cdots>\left(a^{s}, 1, a^{t}\right)<\left(a^{s}, a, a^{t-1}\right)>\left(a^{s+1}, 1, a^{t-1}\right)<\cdots>\left(a^{p}, 1,1\right) .
\end{gathered}
$$

Согласно предложению 1.1 группы гомологий произвольного частично упорядоченного множества $(P, \leqslant)$ с коэффициентами в диаграмме $G: P \rightarrow$ Аb будут изоморфны группам гомологий подкомплекса комплекса $C_{*}(P, G)$, состоящего из абелевых групп $C_{n}^{+}(P, G)=\bigoplus_{p_{0}<\cdots<p_{n}} G\left(p_{0}\right)$, где $p_{0}, \ldots, p_{n}$ - элементы из $P$, удовлетворяющие строгим неравенствам $p_{0}<\cdots<p_{n}$. В случае $G=\Delta \mathbb{Z}$ этот подкомплекс будет состоять из свободных абелевых групп $\bigoplus_{p_{0}<\cdots<p_{n}} \mathbb{Z}$ и дифференциалов, определенных на элементах базисов как

$$
d_{n}\left(p_{0}<\cdots<p_{n}\right)=\sum_{i=0}^{n}(-1)^{i}\left(p_{0}<\cdots<p_{i-1}<p_{i+1}<\cdots<p_{n}\right) .
$$

Отсюда можно получить комплекс для вычисления групп целочисленных гомологий частично упорядоченного множества $\mathfrak{F} T / a^{p}$

$$
0 \rightarrow \mathbb{Z}^{2 p} \stackrel{d_{1}}{\longrightarrow} \mathbb{Z}^{2 p+1} \rightarrow 0
$$


Дифференциал $d_{1}$ имеет матрицу

$$
\left(\begin{array}{cccccc}
-1 & 0 & 0 & \ldots & 0 & 0 \\
1 & 1 & 0 & \ldots & 0 & 0 \\
0 & -1 & -1 & \ldots & 0 & 0 \\
\vdots & \vdots & \vdots & \ddots & \vdots & \vdots \\
0 & 0 & 0 & \ldots & 1 & 1 \\
0 & 0 & 0 & \ldots & 0 & -1
\end{array}\right)
$$

состоящую из $2 p+1$ строк и $2 p$ столбцов. Поскольку для каждого векторастолбца $x \in \mathbb{Z}^{2 p}$ уравнение $d_{1} x=0$ имеет единственное решение $x=0$, то $d_{1}-$ мономорфизм. Отсюда $H_{n}\left(\mathfrak{F} T / a^{p}\right)=0$ при $n \geqslant 1$ и $H_{0}\left(\mathfrak{F} T / a^{p}\right)=\mathbb{Z}$.

СледСтвие 2.2. Пусть $F: \mathfrak{F} \mathbb{N}^{\circ p} \rightarrow \mathrm{Ab}$ - контравариантная натуральная система на бесконечном ииклическом моноиде, $F(1, a): F(a) \rightarrow F(1)$ и $F(a, 1)$ : $F(a) \rightarrow F(1)$ - ее значения на морбизмах $(1, a): 1 \rightarrow a u(a, 1): 1 \rightarrow a$. Тогда $\lim _{\mathfrak{F}} \mathbb{N}^{\text {op }} F=\operatorname{Coker}(F(a, 1)-F(1, a)) u \underline{\lim }_{1}^{\mathfrak{F} \mathbb{N}^{\text {op }}} F=\operatorname{Ker}(F(a, 1)-F(1, a))$.

ДокАЗАТЕЛЬСтво. Применяя предложение 1.2 , приходим к изоморфизму $\left.\lim _{\mathfrak{l}} \mathfrak{F N}^{\mathrm{op}} F \cong \lim _{n}^{\mathfrak{F} T^{\mathrm{op}}} F\right|_{\mathfrak{F} T^{\mathrm{op}}}$. Категория $\mathfrak{F} T^{\mathrm{op}}$ имеет два объекта и два неединичных морфизма $\alpha_{1}=(a, 1)^{\mathrm{op}}: a \rightarrow 1$ и $\alpha_{2}=(1, a)^{\mathrm{op}}: a \rightarrow 1$. Группы гомологий этой категории рассматривались в примере 1.3. Имеют место следующие изоморфизмы: $\left.{\underset{\lim }{\longrightarrow} 0}_{\mathfrak{F} T^{\mathrm{op}}} F\right|_{\mathfrak{F} T^{\mathrm{op}}} \cong \operatorname{Coker}\left(F\left(\alpha_{1}\right)-F\left(\alpha_{2}\right)\right),\left.\lim _{1}^{\mathfrak{F} T^{\mathrm{op}}} F\right|_{\mathfrak{F} T^{\mathrm{op}}} \cong$ $\operatorname{Ker}\left(F\left(\alpha_{1}\right)-F\left(\alpha_{2}\right)\right)$.

\section{2. Категория факторизаций свободного конечно порожденного} коммутативного моноида. Пусть $M$ - моноид; его размерность Лича $\operatorname{Ld} M=\operatorname{hd}(\mathfrak{F} M)^{\text {op }}$ определяется как гомологическая размерность категории, дуальной к категории факторизаций моноида $M$.

Пусть $\mathbb{N}$ - свободный моноид с образующим $a$. Моноид $\mathbb{N}^{n}$ порожден элементами $a_{1}=(a, 1, \ldots, 1), a_{2}=(1, a, 1, \ldots, 1), \ldots, a_{n}=(1, \ldots, 1, a)$. Пусть $T^{n} \subset \mathbb{N}^{n}$ - подмножество, состоящее из конечных произведений $a_{i_{1}} a_{i_{2}} \cdots a_{i_{k}}$, $1 \leqslant i_{1}<i_{2}<\cdots<i_{k} \leqslant n$. Рассмотрим полную подкатегорию категории $\mathfrak{F}^{n}$, имеющую объектами элементы множества $T^{n}$. Обозначим ее через $\mathfrak{F} T^{n}$. Эта подкатегория будет замкнутой.

\section{ЛЕмма 2.3. Вложение $\mathfrak{F} T^{n} \subset \mathfrak{F N}^{n}$ строго коинициалъно.}

ДокАЗАТЕЛьСтво. Для каждого $\alpha=\left(a^{p_{1}}, a^{p_{2}}, \ldots, a^{p_{n}}\right)$ объекты категории $\mathfrak{F} T^{n} / \alpha$ можно рассматривать как морфизмы

$$
\left(a^{\varepsilon_{1}}, a^{\varepsilon_{2}}, \ldots, a^{\varepsilon_{n}}\right) \rightarrow\left(a^{p_{1}}, a^{p_{2}}, \ldots, a^{p_{n}}\right),
$$

где $\varepsilon_{i} \in\{0,1\}$. Ее морфизмам будут соответствовать коммутативные треугольники (рис. 8), где $\varepsilon_{i} \in\{0,1\}, \varepsilon_{i}^{\prime} \in\{0,1\}$, для всех $1 \leqslant i \leqslant n$. Отсюда заключаем, что $\mathfrak{F} T^{n} / \alpha \cong\left(\mathfrak{F} T / a^{p_{1}}\right) \times \cdots \times\left(\mathfrak{F} T / a^{p_{n}}\right)$. По лемме 1.5 категории $\mathfrak{F} T / a^{p_{i}}$ являются частично упорядоченными множествами. Пользуясь следствием 1.15 и леммой 2.1 , устанавливаем, что $H_{q}\left(\mathfrak{F} T^{n} / \alpha\right) \cong H_{q}(p t)$.

СлЕДСТвиЕ 2.4. Верно равенство $\operatorname{Ld} \mathbb{N}^{n}=n$. 


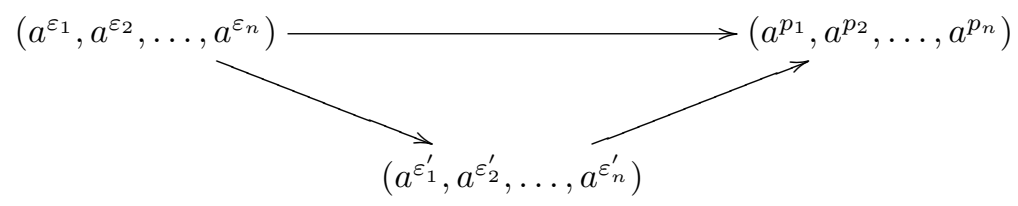

Рис. 8. Морфизмы категории $\mathfrak{F} T^{n} / \alpha$

ДокАЗАТЕЛЬство. Согласно следствию 1.8 имеет место $\mathrm{hd}\left(\mathfrak{F} T^{n}\right)^{\mathrm{op}} \leqslant n$. Отсюда $\operatorname{hd}\left(\mathfrak{F} \mathbb{N}^{n}\right)^{\mathrm{op}} \leqslant n$. Поскольку $\left(\mathfrak{F} \mathbb{N}^{n}\right)^{\mathrm{op}} \cong\left((\mathfrak{F} \mathbb{N})^{\mathrm{op}}\right)^{n}$, то мы можем воспользоваться формулой Кюннета

$$
H_{n}\left(\left((\mathfrak{F} \mathbb{N})^{\mathrm{op}}\right)^{n}, \Delta \mathbb{Z}\right) \cong H_{1}\left((\mathfrak{F} \mathbb{N})^{\mathrm{op}}, \Delta \mathbb{Z}\right)^{\otimes n} \cong \mathbb{Z} .
$$

Следовательно, $\operatorname{hd}\left(\mathfrak{F} \mathbb{N}^{n}\right)^{\text {op }}=n$.

\section{3. Кубические гомологии свободного конечно порожденного ком-} мутативного моноида. Пусть $a_{1}=(a, 1, \ldots, 1), a_{2}=(1, a, 1, \ldots, 1), \ldots, a_{n}=$ $(1, \ldots, 1, a)$ - образующие моноида $\mathbb{N}^{n}$. Для любого слова $a_{i_{1}} \cdots a_{i_{k}}$ и числа $s$, $1 \leqslant s \leqslant k$, обозначим через $a_{i_{1}} \cdots a_{i_{s-1}} \widehat{a_{i_{s}}} a_{i_{s+1}} \cdots a_{i_{k}}$ слово, полученное из него удалением символа $a_{i_{s}}$.

Рассмотрим полукубическое множество $T_{*}^{n}$, состоящее из множеств

$$
T_{k}^{n}=\left\{a_{i_{1}} a_{i_{2}} \cdots a_{i_{k}}: 1 \leqslant i_{1}<i_{2}<\cdots<i_{k} \leqslant n\right\}
$$

и отображений

$$
\begin{gathered}
T_{k-1}^{n} \underset{\partial_{s}^{k, 1}}{\stackrel{\partial_{s}^{k, 0}}{\leftrightarrows}} T_{k}^{n}, \\
\partial_{s}^{k, 0}\left(a_{i_{1}} \cdots a_{i_{k}}\right)=\partial_{s}^{k, 1}\left(a_{i_{1}} \cdots a_{i_{k}}\right)=a_{i_{1}} \cdots a_{i_{s-1}} \widehat{a_{i_{s}}} a_{i_{s+1}} \cdots a_{i_{k}}, \quad 1 \leqslant s \leqslant k .
\end{gathered}
$$

Объекты категории $\square_{+} / T_{*}^{n}$ можно рассматривать как пары $(k, \sigma)$, где $\sigma \in T_{k}^{n}$. Каждый $\sigma \in T_{k}^{n}$ единственным образом записывается в виде $a_{i_{1}} \cdots a_{i_{k}}$, где $1 \leqslant i_{1}<\cdots<i_{k} \leqslant n$. Поэтому объекты $(k, \sigma)$ можно отождествлять с элементами $a_{i_{1}} \cdots a_{i_{k}} \in T^{n}$. Морфизмами в $\square_{+} / T_{*}^{n}$ служат такие тройки $\left(\delta: \mathbb{I}^{m} \rightarrow \mathbb{I}^{k}\right.$, $\left.a_{j_{1}} \cdots a_{j_{m}}, a_{i_{1}} \cdots a_{i_{k}}\right)$, что $T^{n}(\delta)\left(a_{j_{1}} \cdots a_{j_{m}}\right)=a_{i_{1}} \cdots a_{i_{k}}$. Это позволяет построить функтор $\mathfrak{S}: \square_{+} / T_{*}^{n} \rightarrow \mathfrak{F} T^{n}$ следующим образом.

Положим на объектах $\mathfrak{S}\left(a_{i_{1}} \cdots a_{i_{k}}\right)=a_{i_{1}} \cdots a_{i_{k}}$. Функтор $\mathfrak{S}$ определим на морфизмах так:

$$
\begin{aligned}
& \mathfrak{S}\left(\delta_{s}^{k, 0}, a_{i_{1}} \cdots \widehat{a_{i_{s}}} \cdots a_{i_{k}}, a_{i_{1}} \cdots a_{i_{k}}\right)=\left(a_{i_{s}}, 1\right): a_{i_{1}} \cdots \widehat{a_{i_{s}}} \cdots a_{i_{k}} \rightarrow a_{i_{1}} \cdots a_{i_{k}}, \\
& \mathfrak{S}\left(\delta_{s}^{k, 1}, a_{i_{1}} \cdots \widehat{a_{i_{s}}} \cdots a_{i_{k}}, a_{i_{1}} \cdots a_{i_{k}}\right)=\left(1, a_{i_{s}}\right): a_{i_{1}} \cdots \widehat{a_{i_{s}}} \cdots a_{i_{k}} \rightarrow a_{i_{1}} \cdots a_{i_{k}} .
\end{aligned}
$$

Каждый морфизм категории $\square_{+} / T_{*}^{n}$ разлагается в композицию морфизмов вида $\left(\delta_{s}^{k, \varepsilon}, a_{i_{1}} \cdots \widehat{a_{i_{s}}} \cdots a_{i_{k}}, a_{i_{1}} \cdots a_{i_{k}}\right), \varepsilon \in\{0,1\}$. Для всех $1 \leqslant s<t \leqslant k$ и $\alpha, \beta \in\{0,1\}$ имеют место соотношения

$$
\mathfrak{S}\left(\delta_{t}^{k-1, \beta}\right) \mathfrak{S}\left(\delta_{s}^{k, \alpha}\right)=\mathfrak{S}\left(\delta_{s}^{k-1, \alpha}\right) \mathfrak{S}\left(\delta_{t-1}^{k, \beta}\right),
$$


где $\mathfrak{S}(\delta)$ - сокращенное обозначение значения функтора $\mathfrak{S}$ на морфизме $\left(\delta: \mathbb{I}^{m} \rightarrow \mathbb{I}^{k}, a_{j_{1}} \cdots a_{j_{m}}, a_{i_{1}} \cdots a_{i_{k}}\right)$. Отсюда вытекает, что отображение $\mathfrak{S}$, определенное выше на морфизмах $\left(\delta_{s}^{k, \varepsilon}, a_{i_{1}} \cdots \widehat{a_{i_{s}}} \cdots a_{i_{k}}, a_{i_{1}} \cdots a_{i_{k}}\right)$, единственным образом продолжается до функтора. Легко видеть, что значения $\mathfrak{S}(\delta)$ состоят из пар морфизмов $(f, g)$, где $f$ - значение $\mathfrak{S}$ на композиции входящих в каноническое разложение морфизма $\delta$ морфизмов вида $\delta_{s}^{k, 0}$, а $g$ - то же самое для морфизмов вида $\delta_{s}^{k, 1}$.

ЛЕмма 2.5. Функтор $\mathfrak{S}: \square_{+} / T_{*}^{n} \rightarrow \mathfrak{F} T^{n}$ строго коинициален.

ДокАЗАТЕЛЬСтво. Для каждого $\sigma=a_{i_{1}} \cdots a_{i_{k}}$ категория $\mathfrak{S} / \sigma$ имеет терминальный объект и поэтому ациклична.

Произвольной контравариантной натуральной системе $F:\left(\mathfrak{F} \mathbb{N}^{n}\right)^{\mathrm{op}} \rightarrow \mathrm{Ab}$ поставим в соответствие гомологическую систему $\bar{F}=\left.F\right|_{\left(\mathfrak{F} T^{n}\right)^{\text {op }}} \circ \mathfrak{S}^{\text {op }}$ на полукубическом множестве $T_{*}^{n}$.

ТеОРема 2.6. Пусть $F:\left(\mathfrak{F}^{n}\right)^{\mathrm{op}} \rightarrow \mathrm{Ab}$ - контравариантная натуральная система абелевых групп на моноиде $\mathbb{N}^{n}$. Тогда для всех $k \geqslant 0$ имеют место изоморфизмы групп гомологий Лича и кубических групп гомологий,

$$
H_{k}\left(\mathbb{N}^{n}, F\right) \cong H_{k}\left(T_{*}^{n}, \bar{F}\right) .
$$

ДокАЗАТЕЛьСтво. По лемме 2.3 вложение $\mathfrak{F} T^{n} \subset \mathfrak{F} \mathbb{N}^{n}$ строго коинициально и, следовательно, $\left.\underline{\lim }_{\longrightarrow}\left(\mathfrak{F} \mathbb{N}^{n}\right)^{\text {op }} F \cong \lim _{\longrightarrow} F\right|_{\left(\mathfrak{F} T^{n}\right)^{\text {op }}}$.

По лемме 2.5 функтор $\mathfrak{S}$ строго коинициален. Приходим к изоморфизму $\left.\lim _{k}^{\left(\mathfrak{F} \mathbb{N}^{n}\right)^{\mathrm{op}}} F \cong \lim _{k}^{\left(\square_{+} / T_{*}^{n}\right)^{\mathrm{op}}} F\right|_{\left(\mathfrak{F} T^{n}\right)_{\mathrm{op}}} \circ \mathfrak{S}^{\mathrm{op}}$. Определения групп гомологий Лича и кубических групп гомологий вместе с теоремой 1.16 приводят к искомому утверждению.

СЛЕДСТВИЕ 2.7. В условиях теоремъ 2.6 группы гомологий Лича $H_{k}\left(\mathbb{N}^{n}, F\right)$ моноида $\mathbb{N}^{n}$ с коэфбициентами в $F$ будут изоморфны группам $k$ - $x$ гомологий комплекса

$$
\begin{aligned}
& 0 \longleftarrow \\
& \hline \longleftarrow(1) \stackrel{d_{1}}{\longleftarrow} \bigoplus_{1 \leqslant i_{1} \leqslant n} F\left(a_{i_{1}}\right) \stackrel{d_{2}}{\longleftarrow} \bigoplus_{1 \leqslant i_{1}<i_{2} \leqslant n} F\left(a_{i_{1}} a_{i_{2}}\right) \longleftarrow \\
& \cdots \stackrel{d_{n}}{\longleftarrow} F\left(a_{1} a_{2} \cdots a_{n}\right) \longleftarrow 0,
\end{aligned}
$$

где $a_{1}, a_{2}, \ldots, a_{n}$ - образующие моноида $\mathbb{N}^{n} u$

$$
\begin{aligned}
& d_{k}\left(\left(i_{1}, \ldots, i_{k}\right), \varphi\right) \\
& =\sum_{s=1}^{k}(-1)^{s}\left(\left(i_{1}, \ldots, \widehat{i_{s}}, \ldots, i_{k}\right), F\left(a_{i_{1}} \cdots \widehat{a_{i_{s}}} \cdots a_{i_{k}} \stackrel{\left(1, a_{i_{s}}\right)}{\longrightarrow} a_{i_{1}} \cdots a_{i_{k}}\right) \varphi\right. \\
& \left.\quad-F\left(a_{i_{1}} \cdots \widehat{a_{i_{s}}} \cdots a_{i_{k}} \stackrel{\left(a_{i_{s}}, 1\right)}{\longrightarrow} a_{i_{1}} \cdots a_{i_{k}}\right) \varphi\right) .
\end{aligned}
$$

СледСТвИЕ 2.8. Если для любого $1 \leqslant i \leqslant n u \sigma=a_{i_{1}} \cdots a_{i_{k}}, 1 \leqslant i_{1}<i_{2}<$ $\cdots<i_{k} \leqslant n$, имеет место равенство гомоморфизмов

$$
F\left(\left(1, a_{i}\right): \sigma \rightarrow a_{i} \sigma\right)=F\left(\left(a_{i}, 1\right): \sigma \rightarrow a_{i} \sigma\right), \quad i \notin\left\{i_{1}, \ldots, i_{k}\right\},
$$

mo $H_{k}\left(\mathbb{N}^{n}, F\right)=\bigoplus_{1 \leqslant i_{1}<i_{2}<\cdots<i_{k} \leqslant n} F\left(a_{i_{1}} a_{i_{2}} \cdots a_{i_{k}}\right)$.

ДокАЗАТЕЛЬство. В этом случае $d_{k}=0$ для всех $1 \leqslant k \leqslant n$. Это приводит к требуемым изоморфизмам. 
2.4. Категория факторизаций свободного частично коммутативного моноида. Пусть $M(E, I)$ - свободный частично коммутативный моноид с множеством образующих $E$ и множеством определяющих соотношений $a b=b a$, где $(a, b) \in I$. Обозначим через $E_{v} \subseteq E, v \in V$, максимальные подмножества множества образующих моноида $M(E, I)$, состоящие из попарно независимых элементов, а через $M\left(E_{v}\right) \subseteq M(E, I)$ - подмоноид, порожденный множеством $E_{v}$.

Объединение $\bigcup_{v \in V} M\left(E_{v}\right) \subseteq M(E, I)$ не является подкатегорией. Это затрудняет изучение групп гомологий моноида $M(E, I)$. Преодолеть эти трудности помогает идея рассмотреть вложение соответствующих категорий факторизаций: полная подкатегория категории $\mathfrak{F} M(E, I)$, имеющая множество объектов $M\left(E_{v}\right)$, будет совпадать с категорией $\mathfrak{F} M\left(E_{v}\right)$. Более того, $\mathfrak{F} M\left(E_{v}\right) \subseteq$ $\mathfrak{F} M(E, I)$ будет замкнутой.

Изучим сначала комма-категорию $\mathfrak{F} M(E, I) / \alpha$ для произвольного элемента $\alpha \in M(E, I)$.

ПрЕДЛОЖЕНИЕ 2.9. Пусть $M(E, I)$ - свободный частично коммутативный моноид и $\alpha \in M(E, I)$ - произвольный элемент. Тогда категория $\mathfrak{F} M(E, I) / \alpha$ является частично упорядоченным множеством.

ДоказАтельство. Согласно [2; следствие 2] в случае конечного $E$ моноид $M(E, I)$ допускает сокращения. Пусть $E$ бесконечно. Предположим, что $y w_{1} x=y w_{2} x$. Можно подобрать конечное подмножество $E^{\prime} \subseteq E$, содержащее все буквы, входящие в $y, x, w_{1}, w_{2}$. Подмоноид $M\left(E^{\prime}, I \cap\left(E^{\prime} \times E^{\prime}\right)\right)$ допускает сокращения, откуда следует, что $w_{1}=w_{2}$. Стало быть, моноид $M(E, I)$ допускает сокращения. Каждому элементу $x \in M(E, I)$ можно приписать целое число $|x| \geqslant 0$, равное длине слова, представляющего $x$. Это число называется длиной элемента $x$. Для любых $x, y \in M(E, I)$ имеют место равенства $|x y|=|x|+|y|$. Следовательно, моноид $M(E, I)$ не имеет неединичных ретракций. С помощью леммы 1.5 приходим к выводу, что $\mathfrak{F} M(E, I) / \alpha$ - частично упорядоченное множество.

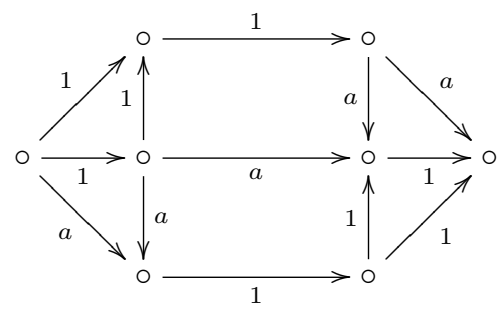

Рис. 9. Морфизмы категории $\mathfrak{F} M(E, I) / a$

ПРИмеР 2.2. Пусть $M(E, I)$ - произвольный свободный частично коммутативный моноид, $a \in E$ - элемент множества образующих. Опишем частично упорядоченное множество $\mathfrak{F} M(E, I) / a$. Оно содержит три элемента: $(1,1, a)$, $(1, a, 1),(a, 1,1)$. Морфизмы между этими элементами определяются коммутативной диаграммой, показанной на рис. 9. Эти морфизмы $(1,1, a) \stackrel{(1, a)}{\longrightarrow}(1, a, 1)$ 
и $(a, 1,1) \stackrel{(a, 1)}{\longrightarrow}(1, a, 1)$ приводят к соотношениям $(1,1, a)<(1, a, 1)>(a, 1,1)$ в частично упорядоченном множестве $\mathfrak{F} M(E, I) / a$.

Элементы $x, y \in M(E, I)$ называются перестановочными, если $x \circ y=y \circ x$. Обратим внимание на то, что образующие $a, b \in E$ перестановочны тогда и только тогда, когда $(a, b) \in I$ либо $a=b$.

Категория $\mathfrak{F} M(E, I)$ содержит полную подкатегорию $\bigcup_{v \in V} \mathfrak{F} M\left(E_{v}\right)$, объектами которой являются произведения попарно перестановочных образующих. Наша следующая задача - изучить комма-категорию $\bigcup_{v \in V} \mathfrak{F} M\left(E_{v}\right) / \alpha$ для $\alpha \in M(E, I)$.

ПримеР 2.3. Пусть $E=\{a, b, c\}, I=\{(a, b),(b, a),(b, c),(c, b)\}, \alpha=a b c$. Категория $\bigcup_{v \in V} \mathfrak{F} M\left(E_{v}\right) / \alpha$ будет частично упорядоченным множеством. На рис. 10 показана диаграмма Хассе частично упорядоченного множества

$$
\bigcup_{v \in V} \mathfrak{F}\left(M\left(E_{v}\right)^{\mathrm{op}}\right) /(a b c) \subset \mathfrak{F}\left(M(E, I)^{\mathrm{op}}\right) /(a b c),
$$

которое согласно следствию 1.4 изоморфно $\bigcup_{v \in V} \mathfrak{F} M\left(E_{v}\right) / \alpha$.

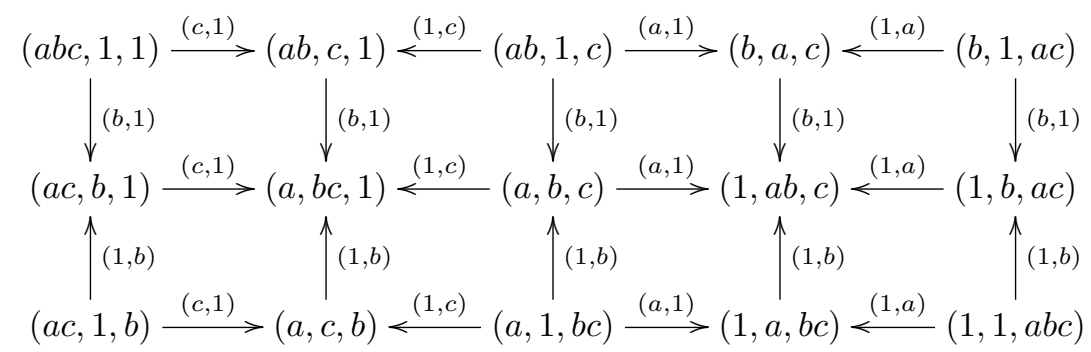

Рис. 10. Категория $\bigcup_{v \in V} \mathfrak{F}\left(M\left(E_{v}\right)^{\mathrm{op}}\right) /(a b c)$

Теорема 2.10. Вложение $\bigcup_{v \in V} \mathfrak{F} M\left(E_{v}\right) \subseteq \mathfrak{F} M(E, I)$ строго коинициалъно.

ДокАЗАтЕЛЬСтво. Согласно лемме 1.3 категория $\mathfrak{F} M(E, I)$ изоморфна категории $\mathfrak{F}\left(M(E, I)^{\mathrm{op}}\right)$. При этом изоморфизме ее подкатегории $\bigcup_{v \in V} \mathfrak{F} M\left(E_{v}\right)$ будет соответствовать подкатегория $\bigcup_{v \in V} \mathfrak{F}\left(M\left(E_{v}\right)^{\mathrm{op}}\right)$. Поэтому достаточно доказать, что вложение $\bigcup_{v \in V} \mathfrak{F}\left(M\left(E_{v}\right)^{\text {op }}\right) \subseteq \mathfrak{F}\left(M(E, I)^{\text {ор }}\right)$ строго коинициально. Это равносильно утверждению о том, что для каждого $\alpha \in M(E, I)$ для всех $q \in \mathbb{N}$ имеют место изоморфизмы

$$
H_{q}\left(\bigcup_{v \in V} \mathfrak{F}\left(M\left(E_{v}\right)^{\mathrm{op}}\right) / \alpha\right) \cong H_{q}(p t) .
$$

Докажем это утверждение с помощью индукции по длине $|\alpha|$. По предложению 2.9 комма-категория $\mathfrak{F}\left(M(E, I)^{\mathrm{op}}\right) / \alpha$ является частично упорядоченным множеством. Согласно следствию 1.4 его элементы можно задавать как тройки $(x, \beta, y)$ элементов моноида $M(E, I)$, для которых $x \circ \beta \circ y=\alpha$. Отношение 
порядка $(x, \beta, y) \leqslant\left(x^{\prime}, \beta^{\prime}, y^{\prime}\right)$ будет определено условием существования элементов $(v, w)$, делающих коммутативной диаграмму, показанную на рис. 3 , т.е. удовлетворяющих соотношениям $x^{\prime} v=x, v \beta w=\beta^{\prime}, w y^{\prime}=y$. Подкатегория $\left(\bigcup_{v \in V} \mathfrak{F}\left(M\left(E_{v}\right)^{\mathrm{op}}\right)\right) / \alpha \cong \bigcup_{v \in V}\left(\mathfrak{F}\left(M\left(E_{v}\right)^{\text {ор }}\right) / \alpha\right)$ будет его замкнутым подмножеством, состоящим из троек $(x, \beta, y)$, для которых $\beta \in \bigcup_{v \in V} M\left(E_{v}\right)$.

Обозначим $\Phi(\alpha)=\bigcup_{v \in V}\left(\mathfrak{F}\left(M\left(E_{v}\right)^{\mathrm{op}}\right) / \alpha\right)$. Докажем по индукции по $|\alpha|$, что частично упорядоченное множество $\Phi(\alpha)$ ациклично. Доказательство основывается на том, что $\Phi(\alpha)$ покрыто замкнутыми подмножествами, изоморфными $\Phi(\beta)$, для элементов $\beta$, имеющих меньшую длину, чем $\alpha$.

Если $|\alpha|=0$, то $\alpha=1$, откуда частично упорядоченное множество $\Phi(\alpha)$ состоит из единственного элемента $(1,1,1)$. Стало быть, в этом случае утверждение верно.

Предположим, что для любого $\beta \in M(E, I)$ при $|\beta|<n$ имеют место изоморфизмы $H_{q}(\Phi(\beta)) \cong H_{q}(p t)$ для всех $q \geqslant 0$. Докажем это для случая, когда $|\alpha|=n$.

Если $\alpha=f \circ g$, то будем называть $f$ левым делителем, а $g$ - правым делителем элемента $\alpha \in M(E, I)$. В этом случае определены инъекции частично упорядоченных множеств

$$
\mathfrak{F}\left(M(E, I)^{\mathrm{op}}\right) / g \stackrel{f_{*}}{\longrightarrow} \mathfrak{F}\left(M(E, I)^{\mathrm{op}}\right) / \alpha \stackrel{g^{*}}{\longleftarrow} \mathfrak{F}\left(M(E, I)^{\mathrm{op}}\right) / f,
$$

где $f_{*}(x, \beta, y)=(f \circ x, \beta, y), g^{*}(x, \beta, y)=(x, \beta, y \circ g)$. Образы отображений

$$
\Phi(g) \stackrel{f_{*}}{\longrightarrow} \Phi(\alpha) \stackrel{g^{*}}{\longleftarrow} \Phi(f)
$$

замкнуты в $\Phi(\alpha)$. В общем случае, если для некоторых $f, g, \gamma \in M(E, I)$ выполнено $f \circ \gamma \circ g=\alpha$, то образ вложения $f_{*} g^{*}=g^{*} f_{*}: \Phi(\gamma) \rightarrow \Phi(\alpha)$ будет замкнут в $\Phi(\alpha)$.

Пусть $\left\{a_{1}, \ldots, a_{m}\right\} \subseteq E-$ множество всех образующих, являющихся левыми делителями элемента $\alpha$, и пусть $\left\{b_{1}, \ldots, b_{n}\right\} \subseteq E$ - множество образующих, которые являются правыми делителями. Легко видеть, что образующие $a_{1}, \ldots, a_{m}$ попарно независимы. Аналогично, $b_{1}, \ldots, b_{n}$ попарно независимы. Обозначим при $1 \leqslant i \leqslant m$ и $1 \leqslant j \leqslant n$

$$
\begin{aligned}
L_{i}(\alpha) & =\left\{(x, \beta, y) \in \Phi(\alpha): a_{i}-\text { левый делитель для } x\right\} \\
R_{j}(\alpha) & =\left\{(x, \beta, y) \in \Phi(\alpha): b_{j}-\text { правый делитель для } y\right\} .
\end{aligned}
$$

Пусть $L(\alpha)=\bigcup_{i=1}^{m} L_{i}(\alpha), R(\alpha)=\bigcup_{j=1}^{n} R_{j}(\alpha)$. Легко видеть, что $L(\alpha)=$ $\left\{(x, \beta, y): \beta \in \bigcup_{v \in V} M\left(E_{v}\right), x \neq 1\right\}, R(\alpha)=\left\{(x, \beta, y): \beta \in \bigcup_{v \in V} M\left(E_{v}\right), y \neq 1\right\}$.

Рассмотрим теперь два случая:

$$
\alpha \in \bigcup_{v \in V} M\left(E_{v}\right) \quad \text { и } \quad \alpha \notin \bigcup_{v \in V} M\left(E_{v}\right) .
$$

В первом случае $\Phi(\alpha)$ содержит наибольший элемент $(1, \alpha, 1)$. В этом случае $H_{q}(\Phi(\alpha)) \cong H_{q}(p t)$ для всех $q \geqslant 0$.

В случае $\alpha \notin \bigcup_{v \in V} M\left(E_{v}\right)$ для каждого элемента $(x, \beta, y) \in \Phi(\alpha)$ имеет место $x \neq 1$ или $y \neq 1$. Следовательно, $(x, \beta, y) \in L(\alpha) \cup R(\alpha)$. В этом случае получаем $\Phi(\alpha)=L(\alpha) \cup R(\alpha)$. 
Воспользуемся леммой 1.12 для доказательства того, что $L(\alpha) \cup R(\alpha)$ ациклично. Если $\alpha=f \circ g$, то обозначим $g$ через $f^{-1} \alpha$, а $f$ - через $\alpha g^{-1}$.

1) Докажем ацикличность $L(\alpha)=\bigcup_{i=1}^{m} L_{i}(\alpha)$. Имеем $L_{i_{1}}(\alpha) \cap \cdots \cap L_{i_{k}}(\alpha)=$ $\left(a_{i_{1}} \cdots a_{i_{k}}\right)_{*} \Phi\left(\left(a_{i_{1}} \cdots a_{i_{k}}\right)^{-1} \alpha\right)$. Отображение $\left(a_{i_{1}} \cdots a_{i_{k}}\right)_{*}$ осуществляет изоморфизм частично упорядоченного множества $\Phi\left(\left(a_{i_{1}} \cdots a_{i_{k}}\right)^{-1} \alpha\right)$ на замкнутое подмножество, откуда следует, что пересечения $L_{i_{1}}(\alpha) \cap \cdots \cap L_{i_{k}}(\alpha)$ ацикличны. С помощью следствия 1.13 получаем ацикличность $L(\alpha)$.

2) Ацикличность $R(\alpha)=\bigcup_{j=1}^{n} R_{j}(\alpha)$ доказывается аналогично.

3) Имеем

$$
\begin{gathered}
L(\alpha) \cap R(\alpha)=\bigcup_{i=1}^{m}\left(L_{i}(\alpha) \cap R(\alpha)\right), \\
L_{i_{1}}(\alpha) \cap \cdots \cap L_{i_{k}}(\alpha) \cap R(\alpha) \\
=\left\{\left(a_{i_{1}} \cdots a_{i_{k}} x, \beta, y\right): \beta \in \bigcup_{v \in V} M\left(E_{v}\right), y \neq 1, a_{i_{1}} \cdots a_{i_{k}} x \beta y=\alpha\right\} .
\end{gathered}
$$

Отсюда следует, что подмножество

$$
L_{i_{1}}(\alpha) \cap \cdots \cap L_{i_{k}}(\alpha) \cap R(\alpha)=\left(a_{i_{1}} \cdots a_{i_{k}}\right)_{*} R\left(\left(a_{i_{1}} \cdots a_{i_{k}}\right)^{-1} \alpha\right)
$$

изоморфно частично упорядоченному множеству $R\left(\left(a_{i_{1}} \cdots a_{i_{k}}\right)^{-1} \alpha\right)$, ацикличность которого установлена в пункте 2).

Применяя лемму 1.12 , приходим к выводу, что $\Phi(\alpha)$ ациклично.

\section{5. Размерность Лича свободного частично коммутативного мо- ноида.}

ТЕОРЕма 2.11. Пусть $M(E, I)$ - свободный частично коммутативный моноид, в котором максимальное число попарно независимых образующих равно $n$. Тогда $\operatorname{Ld} M(E, I)=n$.

ДоказАтельство. Пусть $\bigcup_{v \in V} E_{v}$ - покрытие множества $E$ его максимальными подмножествами попарно независимых элементов, $M\left(E_{v}\right) \subseteq M(E, I)-$ подмоноиды, порожденные множествами $E_{v}$. В силу того, что подкатегория $\bigcup_{v \in V} \mathfrak{F} M\left(E_{v}\right)$ замкнута в $\mathfrak{F} M(E, I)$ по лемме 1.11 верно неравенство

$$
\operatorname{hd}\left(\bigcup_{v \in V} \mathfrak{F} M\left(E_{v}\right)\right)^{\mathrm{op}} \leqslant \operatorname{hd}(\mathfrak{F} M(E, I))^{\mathrm{op}} .
$$

Вложение $\bigcup_{v \in V} \mathfrak{F} M\left(E_{v}\right) \subseteq \mathfrak{F} M(E, I)$ строго коинициально по теореме 2.10 . Применяя следствие 1.9 , приходим к неравенству

$$
\operatorname{hd}\left(\bigcup_{v \in V} \mathfrak{F} M\left(E_{v}\right)\right)^{\mathrm{op}} \geqslant \operatorname{hd}(\mathfrak{F} M(E, I))^{\mathrm{op}} .
$$

Следовательно, $\operatorname{hd}\left(\bigcup_{v \in V} \mathfrak{F} M\left(E_{v}\right)\right)^{\text {op }}=\operatorname{hd}(\mathfrak{F} M(E, I))^{\text {op }}$. Отсюда с помощью леммы 1.11 получаем равенство

$$
\operatorname{hd}(\mathfrak{F} M(E, I))^{\mathrm{op}}=\sup _{v \in V} \operatorname{hd}\left(\mathfrak{F} M\left(E_{v}\right)\right)^{\mathrm{op}}
$$


и вместе с ним $\operatorname{Ld} M(E, I)=\sup _{v \in V} \operatorname{Ld} M\left(E_{v}\right)$. Размерность Лича свободного коммутативного моноида, порожденного $n$ элементами, равна $n$ согласно следствию 2.4. Отсюда заключаем, что $\operatorname{Ld} M(E, I)=n$, где $n$ - максимальное число образующих моноидов $M\left(E_{v}\right)$.

2.6. Гомологическая размерность свободного частично коммутативного моноида. Получаем следующее подтверждение высказанной в [3] гипотезы о гомологической размерности свободного частично коммутативного моноида.

ТеОРема 2.12. Пусть $M(E, I)$ - свободный частично коммутативный моноид, в котором максимальное число попарно независимых образующих равно $n<\infty$. Тогда hd $M(E, I)=n$.

ДокАЗАТЕЛЬСтво. Подставим в лемме $1.6 \mathscr{C}=M(E, I)^{\mathrm{op}}$. Функтор $t$ : $\mathfrak{F}\left(M(E, I)^{\mathrm{op}}\right) \rightarrow M(E, I)^{\mathrm{op}}$ строго коинициален. Категории $\mathfrak{F}\left(M(E, I)^{\mathrm{op}}\right)$ и $\mathfrak{F} M(E, I)$ изоморфны. Получаем строго коинициальный функтор $\mathfrak{F} M(E, I) \rightarrow$ $M(E, I)^{\mathrm{op}}$. Отсюда $\mathrm{hd}(\mathfrak{F} M(E, I))^{\mathrm{op}} \geqslant \mathrm{hd} M(E, I)$. Теорема 2.11 приводит к неравенству hd $M(E, I) \leqslant n$. Докажем равенство. С этой целью выберем максимальное подмножество попарно независимых образующих $E_{v}$, состоящее из $n$ элементов. Так как $M\left(E_{v}\right) \cong \mathbb{N}^{n}$, то с помощью формулы Кюннета легко получить, что $\lim _{n}^{M\left(E_{v}\right)} \Delta \mathbb{Z} \cong \mathbb{Z}$. С помощью леммы 1.6 получим, что $\lim _{\mathfrak{l}} \mathfrak{F} M\left(E_{v}\right)^{\mathrm{op}} \Delta \mathbb{Z} \cong \mathbb{Z}$. Лемма 1.10 приводит к соотношению

$$
\lim _{\mathfrak{l}} \mathfrak{F} M(E, I)^{\mathrm{op}}(\Delta \mathbb{Z})_{\mathfrak{F} M\left(E_{v}\right)^{\mathrm{op}}} \cong \mathbb{Z},
$$

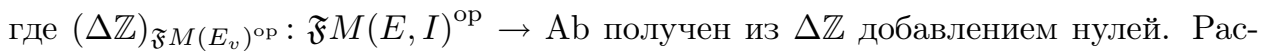
сматривая точную последовательность

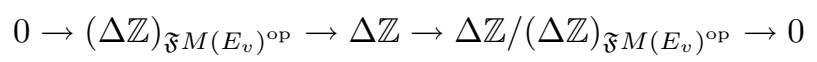

и связанную с ней длинную точную последовательность, получаем мономорфизм $\mathbb{Z} \rightarrow \underset{\lim }{\mathfrak{F} M(E, I)^{\mathrm{op}}} \Delta \mathbb{Z}$. Следовательно,

$$
\lim _{n}^{M(E, I)} \Delta \mathbb{Z} \cong \lim _{n} \mathfrak{F} M(E, I)^{\mathrm{op}} \Delta \mathbb{Z} \neq 0,
$$

откуда hd $M(E, I) \geqslant n$.

Применим эту теорему для оценки гомологической размерности асинхронной системы переходов. Согласно [1] асинхронную систему можно определить как пару $T=(M(E, I), X)$, состоящую из свободного частично коммутативного моноида $M(E, I)$ и правого пунктированного $M(E, I)$-множества $X$. Ей соответствует категория состояний $K_{*}(T)$, объектами которой являются элементы $x \in X$, а морфизмами служат тройки $x \stackrel{\mu}{\rightarrow} y$ элементов $x, y \in X$ и $\mu \in M(E, I)$, удовлетворяющих равенству $x \cdot \mu=y$. Здесь точкой обозначено действие моноида. Поставлена следующая задача: доказать, что если любое подмножество попарно независимых элементов из $E$ имеет не более $n$ элементов, то hd $K_{*}(T) \leqslant n[1 ;$ открытая проблема 2].

СЛЕДСТВИЕ 2.13. Пусть, как и выше, $T=(M(E, I), X)$ - правое пунктированное $M(E, I)$-множество и $n>0$ - максимальное число попарно независимых элементов из $E$. Тогда hd $K_{*}(T) \leqslant n$. 
ДокАЗАТЕЛЬСтво. Максимальное число попарно независимых образующих дуального моноида $M(E, I)^{\text {ор }}$ равно $n$. Из теоремы 2.12 вытекает, что hd $M(E, I)^{\text {op }}=n$. Согласно [1; теорема 5.3] для любого функтора $F: K_{*}(T) \rightarrow$ Ab существует такой функтор $\widetilde{F}: M(E, I)^{\mathrm{op}} \rightarrow \mathrm{Ab}$, что

$$
\lim _{k} K_{*}^{(T)} F \cong \lim _{k}^{M(E, I)^{\mathrm{op}}} \widetilde{F} .
$$

Следовательно, $\lim _{k} K_{*}(T)=0$ для всех $k>n$.

2.7. Гомологии Лича и кубические гомологии свободного частично коммутативного моноида. Упорядочим множество $E$ с помощью некоторого отношения линейного порядка <. Нам понадобится следующее вспомогательное утверждение.

ПРЕДЛОЖЕНИЕ 2.14. Пусть $T_{v} \subset M\left(E_{v}\right)$ для каждого $v \in V$ обозначает подмножество, состоящее из произведений $a_{1} a_{2} \cdots a_{n}$ таких, что $a_{1}<a_{2}<$ $\cdots<a_{n} u a_{j} \in E_{v}$ для всех $1 \leqslant j \leqslant n$. Здесь $n$ может принимать конечные значения, не большие, чем $\left|E_{v}\right|$. Предположим, что при $n=0$ это произведение равно $1 \in T_{v}$. Тогда вложение $\bigcup_{v \in V} \mathfrak{F} T_{v} \subset \mathfrak{F} M(E, I)$ строго коинициально.

ДокАЗАТЕЛЬСтво. Из предложения 1.2 следует, что композиция строго коинициальных функторов строго коинициальна [5; следствие 3.5]. Мы доказали, что вложение $\bigcup_{v \in V} \mathfrak{F} M\left(E_{v}\right) \subseteq \mathfrak{F} M(E, I)$ строго коинициально. Поэтому достаточно доказать, что строго коинициально вложение $\bigcup_{v \in V} \mathfrak{F} T_{v} \subset \bigcup_{v \in V} \mathfrak{F} M\left(E_{v}\right)$. Но для каждого $\alpha \in \bigcup_{v \in V} \mathfrak{F} M\left(E_{v}\right)$ существует такой $w \in V$, что $\alpha \in \mathfrak{F} M\left(E_{w}\right)$. Все делители элемента $\alpha$ будут принадлежать $M\left(E_{w}\right)$. Отсюда $\bigcup_{v \in V} \mathfrak{F} T_{v} / \alpha=$ $\mathfrak{F} T_{w} / \alpha$.

По доказанной выше лемме 2.3 вложение $\mathfrak{F} T_{w} \subset \mathfrak{F} M\left(E_{w}\right)$ строго коинициально. Стало быть, $H_{q}\left(\bigcup_{v \in V} \mathfrak{F} T_{v} / \alpha\right) \cong H_{q}(p t)$.

Перейдем к построению комплекса для вычисления гомологий Лича свободных частично коммутативных моноидов. Для произвольного множества $E$ и антирефлексивного симметричного отношения $I \subseteq E \times E$ построим полукубическое множество $T(E, I)$, зависящее от некоторого отношения линейного порядка $\leqslant$ на $E$. С этой целью для каждого целого $n>0$ определим $T(E, I)_{n}$ как множество слов $a_{1} \cdots a_{n}$, состоящее из попарно перестановочных элементов $a_{1}<\cdots<a_{n}$ множества $E$ :

$$
T(E, I)_{n}=\left\{a_{1} \cdots a_{n}:\left(a_{1}<\cdots<a_{n}\right) \&\left(1 \leqslant i<j \leqslant n \Longrightarrow\left(a_{i}, a_{j}\right) \in I\right)\right\} .
$$

При $n=0$ множество $T(E, I)_{0}$ состоит из пустого слова 1. Отображения $\partial_{i}^{n, \varepsilon}: T(E, I)_{n} \rightarrow T(E, I)_{n-1}$ при $1 \leqslant i \leqslant n$ действуют как

$$
\partial_{i}^{n, 0}\left(a_{1} \cdots a_{n}\right)=\partial_{i}^{n, 1}\left(a_{1} \cdots a_{n}\right)=\left(a_{1} \cdots \widehat{a_{i}} \cdots a_{n}\right) .
$$

Пусть $E=\bigcup_{v \in V} E_{v}$ - разложение множества $E$ в объединение максимальных подмножеств попарно независимых элементов. Тогда $T(E, I)$ равно объединению полукубических подмножеств $\left(T_{v}\right)_{*}$, состоящих из множеств

$$
\left(T_{v}\right)_{n}=\left\{a_{1} \cdots a_{n} \in T(E, I)_{n}: a_{i} \in E_{v} \text { при } 1 \leqslant i \leqslant n\right\} .
$$

Граничные отображения $\left(T_{v}\right)_{n} \stackrel{\partial_{i}^{n, \varepsilon}}{\longrightarrow}\left(T_{v}\right)_{n-1}$ действуют при $1 \leqslant i \leqslant n, \varepsilon \in\{0,1\}$ по формулам (1). Пусть $\mathfrak{S}: \square_{+} / \bigcup_{v \in V}\left(T_{v}\right)_{*} \rightarrow \bigcup_{v \in V} \mathfrak{F} T_{v}-$ функтор, ставящий 
в соответствие каждому сингулярному кубику $a_{1} \cdots a_{n} \in \bigcup_{v \in V}\left(T_{v}\right)_{n}$ объект $a_{1} \cdots a_{n}$. На морфизмах функтор $\mathfrak{S}$ действует по формулам, аналогичным формулам в случае коммутативного моноида.

Для любого функтора $F:(\mathfrak{F} M(E, I))^{\mathrm{op}} \rightarrow \mathrm{Ab}$ обозначим через $\bar{F}$ гомологическую систему на $T(E, I)$, равную композиции

$$
\left(\square_{+} / T(E, I)\right)^{\mathrm{op}} \stackrel{\mathfrak{S}^{\mathrm{op}}}{\longrightarrow} \bigcup_{v \in V}\left(\mathfrak{F} T_{v}\right)^{\mathrm{op}} \subset(\mathfrak{F} M(E, I))^{\mathrm{op}} \stackrel{F}{\longrightarrow} \mathrm{Ab} .
$$

ОПРЕДЕЛЕНИЕ 2.4. Свободный частично коммутативный моноид $M(E, I)$ называется локалъно конечномерным, если любое подмножество множества $E$, состоящее из попарно перестановочных элементов, имеет конечное число элементов.

Ниже везде будем предполагать, что на множестве $E$ задано некоторое отношение линейного порядка и множества $T(E, I)_{n}$ состоят из произведений $a_{1} \cdots a_{n}$ попарно независимых элементов $a_{1}<\cdots<a_{n}$ из $E$.

ТеОРема 2.15. Пусть $M(E, I)$ - локально конечномерный свободный ча-

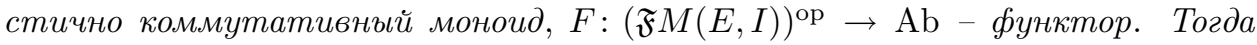
для всех $n \geqslant 0$ имеют место изоморфизмы групп гомологий Лича и кубических групп гомологий,

$$
H_{n}(M(E, I), F) \cong H_{n}(T(E, I), \bar{F}) .
$$

ДокАЗАТЕЛЬСтво. Образ ограничения функтора $\mathfrak{S}$ на подкатегорию $\square_{+} /\left(T_{v}\right)_{*}$ содержится в категории $\mathfrak{F} T_{v}$ и определяет функтор, который мы обозначим через $\mathfrak{S}_{v}: \square_{+} /\left(T_{v}\right)_{*} \rightarrow \mathfrak{F} T_{v}$. Для произвольного $\alpha \in \bigcup_{v \in V} \mathfrak{F} T_{v}$ существует такой $w \in V$, что $\alpha \in \mathfrak{F} T_{w}$. Отсюда следует, что будет иметь место изоморфизм $\mathfrak{S} / \alpha \cong \mathfrak{S}_{w} / \alpha$. По лемме 2.5 для свободного конечно порожденного коммутативного моноида $M\left(E_{v}\right)$ функтор $\mathfrak{S}_{v}: \square_{+} /\left(T_{v}\right)_{*} \rightarrow \mathfrak{F} T_{v}$ строго коинициален. Стало быть, категория $\mathfrak{S}_{v} / \alpha$ ациклична. Вместе с ней категория $\mathfrak{S} / \alpha$ ациклична, а функтор $\mathfrak{S}$ - строго коинициален. Пользуясь тем, что вложение $\bigcup_{v \in V} \mathfrak{F} T_{v} \subseteq \mathfrak{F} M(E, I)$ строго коинициально, приходим к доказываемым изоморфизмам

$$
\lim _{n}(\mathfrak{F} M(E, I))^{\mathrm{op}} F \cong \lim _{\longrightarrow}\left(\square_{+} / \bigcup_{v \in V}\left(T_{v}\right)_{*}\right)^{\mathrm{op}}\left(\left.F\right|_{\bigcup_{v \in V}\left(\mathfrak{F} T_{v}\right)^{\mathrm{op}}} \circ \mathfrak{S}^{\mathrm{op}}\right) .
$$

Отсюда получаем следующий основной результат работы.

ТеОРема 2.16. Пусть $M(E, I)$ - локально конечномерный свободный ча-

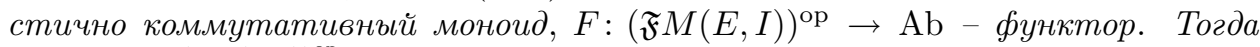
группь $\lim _{\longrightarrow}^{(\mathfrak{F} M(E, I))^{\mathrm{op}}} F$ изоморфны группам гомологий комплекса

$$
\begin{aligned}
& 0 \longleftarrow F(1) \stackrel{d_{1}}{\longleftarrow} \bigoplus_{a_{1} \in E} F\left(a_{1}\right) \stackrel{d_{2}}{\longleftarrow} \bigoplus_{a_{1} a_{2} \in T(E, I)_{2}} F\left(a_{1} a_{2}\right) \longleftarrow \\
& \cdots \longleftarrow \bigoplus_{a_{1} \cdots a_{n-1} \in T(E, I)_{n-1}} F\left(a_{1} \cdots a_{n-1}\right) \stackrel{d_{n}}{\longleftarrow} \bigoplus_{a_{1} \cdots a_{n} \in T(E, I)_{n}} F\left(a_{1} \cdots a_{n}\right) \longleftarrow \cdots
\end{aligned}
$$


$n$-й член которого при $n \geqslant 0$ равен прямой сумме абелевых групп $F\left(a_{1} \cdots a_{n}\right)$ по всем последовательностям попарно перестановочных элементов $a_{1}<a_{2}<$ $\cdots<a_{n}$ множества $E$, а дифференциал определен по формуле

$$
d_{n}\left(a_{1} \cdots a_{n}, \varphi\right)=\sum_{i=1}^{n}(-1)^{i}\left(a_{1} \cdots \widehat{a_{i}} \cdots a_{n}, F\left(1, a_{i}\right) \varphi-F\left(a_{i}, 1\right) \varphi\right),
$$

где $F\left(1, a_{i}\right)$ - значение $F$ на морфизме $\left(1, a_{i}\right): a_{1} \cdots \widehat{a_{i}} \cdots a_{n} \rightarrow a_{1} \cdots a_{n}$, a $F\left(a_{i}, 1\right)$ - на морфизме $\left(a_{i}, 1\right): a_{1} \cdots \widehat{a_{i}} \cdots a_{n} \rightarrow a_{1} \cdots a_{n}$.

ДокАЗАтельСтво. По теореме $2.15 \quad \lim _{\mathfrak{l}}^{(\mathfrak{F} M(E, I))^{\mathrm{op}}} F \cong \lim _{\longrightarrow}^{\left(\square_{+} / T(E, I)\right)^{\mathrm{op}}} \bar{F}$. Применение теоремы 1.16 приводит к изоморфизмам

$$
\lim _{n}^{\mathfrak{F} M(E, I)^{\mathrm{op}}} F \cong H_{n}\left(C_{*}(T(E, I), \bar{F})\right) .
$$

СлеДСтвиЕ 2.17. Пусть $n>0$ - натуральное число и $F$ - контравариантная натуральная система конечно порожденных абелевых групп на $M(E, I)$. Если множество $\left\{\left(a_{1}, \ldots, a_{n}\right): 1 \leqslant i<j \leqslant n \Longrightarrow\left(a_{i}, a_{j}\right) \in I\right\}$ конечно, то для каждого $k>n$ группа $H_{k}(M(E, I), F)$ конечно порождена.

Если значениями натуральной системы $F$ являются свободные конечно порожденные абелевы группы $F(\alpha)=\mathbb{Z}^{n_{\alpha}}$ и гомоморфизмы заданы с помощью целочисленных матриц $\mathbb{Z}^{n_{\alpha}} \rightarrow \mathbb{Z}^{n_{\beta}}$, то дифференциалы $d_{k}$ при $k>n$ будут задаваться конечными матрицами. При $k>n$ получаем алгоритм вычисления групп $H_{k}(M(E, I), F)$ с помощью приведения этих матриц к нормальной форме Смита.

Теорема 2.16 приводит к комплексу для вычисления групп гомологий с коэффициентами в правом $M(E, I)$-модуле, полученному в работе [16] для случая конечно порожденного моноида $M(E, I)$.

СлЕДСТвИЕ 2.18. Пусть $M(E, I)$ - локально конечномерный свободный частично коммутативный моноид, $G$ - правый $M(E, I)$-модуль. Тогда группь $H_{n}\left(M(E, I)^{\mathrm{op}}, G\right)$ изоморфны группам гомологий комплекса

$$
\begin{aligned}
0 \longleftarrow & G \stackrel{d_{1}}{\longleftarrow} \bigoplus_{a_{1} \in E} G \stackrel{d_{2}}{\longleftarrow} \bigoplus_{a_{1} a_{2} \in T(E, I)_{2}} G \longleftarrow \\
\cdots \longleftarrow & \bigoplus_{a_{1} a_{2} \cdots a_{n-1} \in T(E, I)_{n-1}} G \stackrel{d_{n}}{\longleftarrow} \bigoplus_{a_{1} a_{2} \cdots a_{n} \in T(E, I)_{n}} G \longleftarrow \cdots,
\end{aligned}
$$

$n$-й член которого при $n \geqslant 0$ равен прямой сумме абелевых групп $G$ по всем последовательностям попарно перестановочных элементов $a_{1}<a_{2}<\cdots<a_{n}$ множества E, а дифференииал определен по формуле

$$
d_{n}\left(a_{1} \cdots a_{n}, g\right)=\sum_{i=1}^{n}(-1)^{i}\left(a_{1} \cdots \widehat{a_{i}} \cdots a_{n}, g a_{i}-g\right),
$$

где ga $_{i} \in G$ обозначает результат действия $a_{i}$ на $g \in G$.

ДоказАтельство. По лемме 1.6 функтор $t: \mathfrak{F} M(E, I) \rightarrow M(E, I)$ строго коинициален. Стало быть, группы $H_{n}\left(M(E, I)^{\mathrm{op}}, G\right)$, которые по определению равны $\lim _{n}^{M(E, I)^{\mathrm{op}}} G$, будут изоморфны $\lim _{n}^{\mathfrak{F} M(E, I)^{\mathrm{op}}} G \circ t^{\mathrm{op}}$. Подставляя $t\left(1, a_{i}\right)=a_{i}$ и $t\left(a_{i}, 1\right)=1, G \circ t^{\text {ор }}\left(1, a_{i}\right)(g)=g a_{i}$ и $G \circ t^{\text {ор }}\left(a_{i}, 1\right)(g)=1$, из теоремы 2.16 получаем искомый комплекс. 
В частности, пользуясь тем, что для произвольного моноида $M$ его моноидное кольцо $\mathbb{Z}[M]$ будет проективным правым $M$-модулем, приходим к резольвенте, полученной для случая конечного $E$ в работе [4].

СЛЕДСТВИЕ 2.19. Пусть $M(E, I)$ - локально конечномерный свободный частично коммутативный моноид. Свободные левые модули

$$
P_{0}=\mathbb{Z}[M(E, I)], \ldots, P_{n}=\bigoplus_{a_{1} \cdots a_{n} \in T(E, I)_{n}} \mathbb{Z}[M(E, I)], \ldots
$$

над кольиом $\mathbb{Z}[M(E, I)]$ вместе с гомоморфизмами

$$
0 \longleftarrow \mathbb{Z} \leftarrow P_{0} \stackrel{\partial_{1}}{\longleftarrow} P_{1} \stackrel{\partial_{2}}{\longleftarrow} P_{2} \longleftarrow \cdots,
$$

определенными на образующих как

$$
\partial_{n}\left(a_{1} \cdots a_{n}\right)=\sum_{i=1}^{n}(-1)^{i}\left(a_{i}-1\right) a_{1} \cdots a_{i-1} a_{i+1} \cdots a_{n}
$$

составляют проективную резольвенту $M(E, I)$-модуля $\Delta_{M(E, I)} \mathbb{Z}$.

ДокАЗАтельство. Рассмотрим $\mathbb{Z}[M(E, I)]$ как правый $M(E, I)$-модуль. Поскольку он проективен, то $H_{n}\left(M(E, I)^{\mathrm{op}}, \mathbb{Z}[M(E, I)]\right)=0$ при $n>0$. Фактормножество множества $M(E, I)$ по действию моноида состоит из единственного элемента, откуда нулевая группа гомологий равна $\mathbb{Z}$. Следствие 2.18 приводит к точной последовательности

$$
0 \longleftarrow \mathbb{Z} \longleftarrow \mathbb{Z}[M(E, I)] \stackrel{d_{1}}{\longleftarrow} \bigoplus_{a_{1} \in E} \mathbb{Z}[M(E, I)] \stackrel{d_{2}}{\longleftarrow} \bigoplus_{a_{1} a_{2} \in T(E, I)_{2}} \mathbb{Z}[M(E, I)] \longleftarrow \cdots
$$

Дифференциалы $d_{n}$ этой последовательности перестановочны с левым действием моноида $M(E, I)$. Рассматривая $d_{n}$ как гомоморфизмы левых $M(E, I)$-модулей, мы получаем из них гомоморфизмы $\partial_{n}$.

В заключение отметим следующее. Группы гомологий $M$-модуля являются частным случаем групп гомологий Лича. Тем не менее, как показано в настоящей работе, размерность Лича свободных частично коммутативных моноидов легче поддается оценке, чем обычная гомологическая размерность. Это позволило ответить на вопросы, которые не могли быть решены прямыми методами. На основе этой идеи был построен комплекс для вычисления групп гомологий Лича свободного частично коммутативного моноида, который привел к комплексу для вычисления групп гомологий моноида $M(E, I)$ с коэффициентами в правом $M(E, I)$-модуле.

\section{Список литературы}

[1] A. A. Husainov, "On the homology of small categories and asynchronous transition systems", Homology Homotopy Appl., 6:1 (2004), 439-471.

[2] V. Diekert, Y. Métivier, "Partial commutation and traces", Handbook of formal languages, vol. 3, Springer-Verlag, Berlin, 1997, 457-533.

[3] A. A. Husainov, "On the homology of monoids and distributed systems", 5th International Algebraic Conference in Ukraine, Abstracts, Odessa, 2005. 
[4] Л. Ю. Полякова, "Резольвенты для свободных частично коммутативных моноидов", Сиб. матем. журн., 48:6 (2007), 1295-1304; англ. пер.: L. Yu. Polyakova, "Resolutions for free partially commutative monoids", Siberian Math. J., 48:6 (2007), $1038-1045$.

[5] А.А. Хусаинов, "О группах гомологий полукубических множеств", Сиб. матем. журн., 49:1 (2008), 224-237; англ. пер.: A. A. Khusainov, "Homology groups of semicubical sets", Sib. Math. J., 49:1 (2008), 180-190.

[6] П. Габриель, М. Цисман, Категории частных и теория гомотопий, Мир, М., 1971; пер. с англ.: P. Gabriel, M. Zisman, Calculus of fractions and homotopy theory, Ergebnisse der Mathematik und ihrer Grenzgebiete, 35, Springer-Verlag, Berlin-Heidelberg-New York, 1967.

[7] А.А. Хусаинов, "Сравнение размерностей малой категории", Сиб. матем. журн., 38:6 (1997), 1413-1426; англ. пер.: A. A. Khusainov, "Comparing dimensions of a small category", Siberian Math. J., 38:6 (1997), 1230-1240.

[8] U. Oberst, "Homology of categories and exactness of direct limits", Math. Z., 107:2 (1968), 87-115.

[9] H.-J. Baues, G. Wirsching, "Cohomology of small categories", J. Pure Appl. Algebra, 38:2-3 (1985), 187-211.

[10] B. Mitchell, "A remark on projectives in functor categories", J. Algebra, 69:1 (1981), $24-31$.

[11] С. Маклейн, Гомология, Мир, М., 1966; пер. с англ.: S. MacLane, Homology, Die Grundlehren der mathematischen Wissenschaften, 114, Academic Press, New York; Springer-Verlag, Berlin-Göttingen-Heidelberg, 1963.

[12] T. Kaczynski, K. Mischaikow, M. Mrozek, Computational homology, Appl. Math. Sci., 157, Springer-Verlag, New York, 2004.

[13] E. Goubault, The geometry of concurrency, Thesis, Ecole Normale Supérieure, 1995.

[14] С. Маклейн, Категории для работающего математика, Физматлит, М., 2004; пер. с англ.: S. MacLane, Categories for the working mathematician, Grad. Texts in Math., 5, Springer-Verlag, New York, 1998.

[15] J. Leech, "Cohomology theory for monoid congruences", Houston J. Math., 11:2 (1985), 207-223.

[16] А.А. Хусаинов, В.Е. Лопаткин, И.А. Трещев, "Исследование математической модели параллельных вычислительных процессов методами алгебраической топологии", Сиб. журн. индустр. матем., 11:1 (2008), 141-151.

\section{А. А. Хусаинов (А. А. Khusainov)}

Комсомольский-на-Амуре

государственный технический университет

E-mail: husainov@knastu.ru
Поступила в редакцию 28.04 .2008 